\title{
Spectral Line Survey toward Molecular Clouds in the Large Magellanic Cloud
}

\author{
Yuri Nishimura ${ }^{1}$, Takashi Shimonishi ${ }^{2,3}$, Yoshimasa Watanabe ${ }^{1}$, Nami Sakai $^{4}$, \\ Yuri Aikawa $^{5}$, Akiko Kawamura ${ }^{6}$, and Satoshi Yamamoto ${ }^{1}$
}

Received —

Not to appear in Nonlearned J., 45.

\footnotetext{
${ }^{1}$ Department of Physics, the University of Tokyo, 7-3-1, Hongo, Bunkyo-ku, Tokyo, 1130033, Japan

${ }^{2}$ Frontier Research Institute for Interdisciplinary Sciences, Tohoku University, Aramakiazaaoba 6-3, Aoba-ku, Sendai, Miyagi, 980-8578, Japan

${ }^{3}$ Astronomical Institute, Tohoku University, Aramakiazaaoba 6-3, Aoba-ku, Sendai, Miyagi, 980-8578, Japan

${ }^{4}$ RIKEN, 2-1 Hirosawa, Wako, Saitama 351-0198, Japan

${ }^{5}$ Center for Computational Sciences, The University of Tsukuba, 1-1-1, Tennodai, Tsukuba, Ibaraki 305-8577, Japan

${ }^{6}$ National Astronomical Observatory of Japan, Osawa, Mitaka, Tokyo, 181-8588, Japan
} 


\begin{abstract}
Spectral line survey observations of 7 molecular clouds in the Large Magellanic Cloud (LMC) have been conducted in the $3 \mathrm{~mm}$ band with the Mopra $22 \mathrm{~m}$ telescope to reveal chemical compositions in low metallicity conditions. Spectral lines of fundamental species such as $\mathrm{CS}, \mathrm{SO}, \mathrm{CCH}, \mathrm{HCN}, \mathrm{HCO}^{+}$, and $\mathrm{HNC}$ are detected in addition to those of $\mathrm{CO}$ and ${ }^{13} \mathrm{CO}$, while $\mathrm{CH}_{3} \mathrm{OH}$ is not detected in any source and $\mathrm{N}_{2} \mathrm{H}^{+}$is marginally detected in two sources. The molecular-cloud scale (10 pc scale) chemical composition is found to be similar among the 7 sources regardless of different star formation activities, and hence, it represents the chemical composition characteristic to the LMC without influences of star formation activities. In comparison with chemical compositions of Galactic sources, the characteristic features are (1) deficient N-bearing molecules, (2) abundant $\mathrm{CCH}$, and (3) deficient $\mathrm{CH}_{3} \mathrm{OH}$. The feature (1) is due to a lower elemental abundance of nitrogen in the LMC, whereas the features (2) and (3) seem to originate from extended photodissociation regions and warmer temperature in cloud peripheries due to a lower abundance of dust grains in the low metallicity condition. In spite of general resemblance of chemical abundances among the seven sources, the $\mathrm{CS} / \mathrm{HCO}^{+}$and $\mathrm{SO} / \mathrm{HCO}^{+}$ratios are found to be slightly higher in a quiescent molecular cloud. An origin of this trend is discussed in relation to possible depletion of sulfur along molecular cloud formation.
\end{abstract}

Subject headings: galaxies: individual(The Large Magellanic Cloud) — galaxies: ISM — ISM: molecules — Magellanic Clouds 


\section{Introduction}

The Large Magellanic Cloud (LMC) is the nearest external galaxy $(d=49.97 \pm 1.11$ kpc) (Pietrzyński et al. 2013). Taking advantage of proximity to the Sun, extensive studies have been carried out on molecular-cloud evolution and star formation in a galactic scale (Fukui \& Kawamura (2010) and references therein). In contrast to Galactic molecular clouds, individual distances to molecular clouds in the LMC are almost the same, which guarantees a fixed spatial resolution and thus provides an excellent opportunity for statistical studies of molecular clouds. The LMC is also interesting from an astrochemical point of view. The metallicity in the LMC is known to be lower than in the solar neighborhood by a factor 2 or more (Westerlund 1997). In particular, Dufour et al. (1982) reported that the

$\mathrm{C} / \mathrm{H}$ and $\mathrm{N} / \mathrm{H}$ ratios are lower than those in the solar neighborhood by a factor of 6 and 10, respectively (Table 1). These differences of the elemental abundances significantly should affect physical and chemical processes in molecular clouds. For instance, photodissociation and photoionization are expected to be more effective for a given $\mathrm{H}_{2}$ column density owing to a lower abundance of dust grains in the low metallicity condition (e.g. Millar \& Herbst 1990). Such effects may result in chemical compositions of molecular clouds characteristic to the LMC.

So far, chemical compositions of molecular clouds in the LMC were mainly studied toward high-mass star-forming regions containing well-developed H II regions, such as N113 and N159. Bright molecular emission lines were observed in these sources, and abundances of various molecules derived from such observations are compared with those in high-mass star forming regions in our Galaxy, such as Orion KL and Sgr B2 (Wang et al. 2009; Heikkilä et al. 1999; Chin et al. 1997; Paron et al. 2014). However, the above sources in the LMC involve a cluster of high-mass stars and have a very complex physical structure, which prevents us from understanding intrinsic chemical nature of molecular clouds specific to the 
LMC. For this purpose, we need to observe relatively quiescent molecular clouds without strong influence of cluster formation. Such an effort reported so far is the chemical study of the high-mass star-forming cloud, $\mathrm{N} 44 \mathrm{C}$, toward which several molecules other than $\mathrm{CO}$ and their isotopologues are detected (Chin et al. 1997). However, we still need to observe more sources in order to examine the effect of star-formation activities on chemistry and to extract the intrinsic chemical nature.

Currently available single-dish telescopes located in the southern hemisphere have a typical angular resolution of $30-40$ arcsec in the $3 \mathrm{~mm}$ band, which corresponds to a linear size of $\sim 10 \mathrm{pc}$ at the distance of the LMC. In the single-dish observations, we thus look at the chemical composition averaged over the molecular-cloud scale. Hence, the effect of individual star-forming region on the averaged chemical composition is expected to be smeared out. This is particularly true in the $3 \mathrm{~mm}$ band observations, because molecular line-emission in this band would be dominated by extended molecular gas. This is in contrast to the submillimeter-wave line emission which preferentially traces hot and dense parts of star-forming regions. If so, we will be able to extract the intrinsic chemical composition specific to the LMC with the single-dish observation in the $3 \mathrm{~mm}$ band. Importance of such molecular-cloud scale chemical composition is now being recognized in studies on external galaxies (e.g. Watanabe et al. 2014). The results for the LMC will be a fundamental base for interpreting chemical compositions of distant galaxies observed at a high angular resolution.

With this in mind, we conducted 3-mm spectral line survey observations toward 7 molecular clouds without infrared point sources in the LMC having different star-formation activities by using the Mopra $22 \mathrm{~m}$ telescope. 


\section{Source Selection}

For the spectral line survey, we first selected candidate sources having different star-formation activities. We selected 16 sources on the basis of the CO map data (MAGMA; Wong et al. (2011)), which are not associated with infrared sources ('starless clouds'). Non-detection of the point sources at $8 \mu \mathrm{m}$ and $24 \mu \mathrm{m}$ with Spitzer or AKARI generally means absence of high-mass young stellar objects except for very infant ones (Whitney et al. 2008; Kato et al. 2012; Gruendl \& Chu 2009). These 'starless' sources are referred to hereafter as Category A sources in this paper. We also selected 17 high-mass star forming clouds which are associated with high-mass YSOs observed with Spitzer or $A K A R I$, but not embedded in a prominent $\mathrm{H}$ II regions (referred to hereafter Category $\mathrm{B}$ sources) (Shimonishi et al. 2010, 2013; Seale et al. 2009; Gruendl \& Chu| 2009).

We then observed these candidate sources in the ${ }^{13} \mathrm{CO}(J=1-0), \operatorname{HCO}^{+}(J=1-0)$, and $\operatorname{HCN}(J=1-0)$ lines with the Mopra $22 \mathrm{~m}$ telescope. As a result, we found the bright sources in the $\mathrm{HCO}^{+}$and $\mathrm{HCN}$ line for each category; CO Peak 1 and NQC2 for Category A and N79, N44C, and N11B for Category B. All of the target regions are associated with relatively strong ${ }^{12} \mathrm{CO}(J=1-0)$ emission. Based on the MAGMA CO map of the LMC, a typical CO integrated intensity of the regions such as N159W, N113, and N44BC, in which multiple molecular emission lines were detected by the SEST $15 \mathrm{~m}$ telescope, is $16-61 \mathrm{~K}$ $\mathrm{km} \mathrm{s}^{-1}$. The typical CO integrated intensity of our target sources is $10-34 \mathrm{~K} \mathrm{~km} \mathrm{~s}^{-1}$, which is almost comparable to the above well-studied sources. Hence, the selected sources are likely molecular rich. We will publish details of the source selection and the result of the ${ }^{13} \mathrm{CO}, \mathrm{HCO}^{+}$, and $\mathrm{HCN}$ survey in a separate publication (Shimonishi et al. in prep.).

In addition, we included the two active star forming regions with extended H II regions, N113 and N159W (referred to hereafter Category C sources), as references, in our source list. In total, for this study we selected 7 sources, as summarized in Table 2, Figure 1 shows 
the MAGMA CO $(J=1-0)$ maps and the $24 \mu \mathrm{m}$ images by Spitzer MIPS of the target sources. A brief description of each source is given below, where the cloud name is based on Henize (1956), if available.

\section{(Category A)}

CO Peak 1 is located at the eastern part of the LMC. It does not host any infrared sources, as shown in Figure 1, suggesting that high-mass star formation has not started yet in this cloud. Strong CO $(J=1-0)$ emission $\left(31.1 \mathrm{~K} \mathrm{~km} \mathrm{~s}^{-1}\right)$ is detected in the MAGMA observations. Deep molecular line observations are carried out for the first time in this study.

NQC2 is a quiescent molecular cloud located at the central part of the LMC. This source was recognized in the Herschel PACS map of the LMC, and was confirmed to have fairly strong emission of $\mathrm{HCO}^{+}$during the above survey (Shimonishi et al. in prep.). It also shows a bright CO emission in the MAGMA observations, while it does not harbor $24 \mu \mathrm{m}$ point sources (Figure 1). No molecular line observations have been reported for this source except for that of $\mathrm{CO}$.

\section{(Category B)}

N79 is an H II region located in the western part of the LMC. In this study, we observed the relatively quiescent cloud not directly associated with the H II region. It harbors an embedded high-mass YSO which show dust and ice absorption bands source (Seale et al. 2009).

$\mathrm{N} 44 \mathrm{C}$ is a molecular cloud located in the central part of the LMC. N44C hosts an embedded high-mass YSO named ST2, whose luminosity is estimated to be $\sim 2 \times 10^{5} L_{\odot}$ (Shimonishi et al. 2010). Although a few faint YSOs are involved within the observed beam, the total mid-infrared flux toward the N44C region is dominated by the single high-mass YSO, as shown in Figure 1. Hence, we selected this position as the Category B source. 
Detection of the dust and ice absorption bands in the infrared spectrum of this central YSO (Shimonishi et al. 2010) suggests that the N44C cloud is still in the very early stage of star formation. Note that molecular line observations are observed toward N44BC by Chin et al. (1997), which is separated by $1^{\prime}$ from our observed position and is not associated with high-mass YSOs. In N44BC, the lines of 8 species including $\mathrm{HCO}^{+}, \mathrm{HCN}, \mathrm{HNC}, \mathrm{CCH}$, $\mathrm{CS}$, and SO are detected in the $3 \mathrm{~mm}$ band with the SEST $15 \mathrm{~m}$ telescope. Hence, we can compare our result toward $\mathrm{N} 44 \mathrm{C}$ with theirs to examine the effect of star formation on the chemical composition.

N11B is a member of N11 complex, the second largest H II region after the 30 Doradus region. We observed the position not directly associated with the extended H II region. It involves a relatively evolved YSO, which shows emission lines due to PAH and ionized gas (Seale et al. 2009). Barbá et al. (2003) also reported several embedded YSOs. It is also reported that the $\mathrm{CH}_{3} \mathrm{OH}$ maser is associated with this YSO (Ellingsen et al. 2010). Furthermore, a variety of far-infrared emission lines due to ionized gas are detected toward our beam position by Herschel (Lebouteiller et al. 2012). The above characteristics suggest that the N11B region is relatively evolved in comparison with the other two target sources in this category.

\section{(Category C)}

N113 is an active cluster-forming site located in the central port of the LMC, which aparts from the 30 Doradus region by about $2^{\circ}$. It is associated with prominent $\mathrm{H}$ II regions (NGC1874, NGC 1876, and NGC1877) (Bica et al. 1992). This is a well studied source in astrochemistry; several molecular-line studies have been reported (e.g. Paron et al. 2014; Wang et al. 2009, Chin et al. 1997).

N159W is one of active cluster-forming regions associated with the H II region LH105 (Lucke \& Hodge 1970). This source has extensively been studied as a prominent on-going 
star forming region by observations in various wavelength (e.g. Mizuno et al. 2010; Ott et al. 2010). Molecular line observations are also reported by Heikkilä et al. (1999); Johansson et al. (1994).

\section{Observations}

The spectral line survey observations were carried out with the Mopra $22 \mathrm{~m}$ telescope of the Australia Telescope National Facility (ATNF) from June to October in 2013 and from April to October in 2014. We observed the 7 sources selected above, whose positions are summerized in Table 2, We used the $3 \mathrm{~mm}$ InP HEMT Monolithic Microwave Integrated Circuit (MMIC) receiver as a front end. The observed frequency range is from $85 \mathrm{GHz}$ to $116 \mathrm{GHz}$. We observed two orthogonal polarization signals simultaneously. For NQC2, N79, and N113, the frequency range from $101 \mathrm{GHz}$ to $108 \mathrm{GHz}$ is missing. We did not observe this frequency range for these three sources to save the telescope time, because no spectral lines were detected in the other sources (Table 3). The half-power beam width (HPBW) of the telescope is $38^{\prime \prime}$ and $30^{\prime \prime}$ at 90 and $115 \mathrm{GHz}$, respectively. The telescope pointing was checked by observing nearby $\mathrm{SiO}$ maser source (R Dor) every 1.5 hours, and the pointing accuracy was estimated to be better than $5^{\prime \prime}$. We used the Mopra spectrometer (MOPS) in the 'wideband' mode, which simultaneously cover the band width of $8 \mathrm{GHz}$, as a back end. The frequency resolution is $270 \mathrm{kHz}$ per channel, and we binded 3 successive channels in the analysis to improve the signal-to-noize ratio. The resultant velocity resolution is $2.73 \mathrm{~km}$ $\mathrm{s}^{-1}$ at $90 \mathrm{GHz}$. We employed the position-switching mode (OFF-ON-ON-OFF sequences) with individual integrations of $30 \mathrm{~s}$ for all the observations.

The total on-source integration time was 16, 25, 8, 17, and 26 hours for CO Peak 1, NQC2, N79, N44C, and N11B respectively. As for the active star-forming regions, N113 and N159W, we conducted shorter observations. A typical system temperatures were 200 
$\mathrm{K}$ and $600 \mathrm{~K}$ at $90 \mathrm{GHz}$ and $115 \mathrm{GHz}$, respectively. A typical rms noize temperature in the main-beam temperature scale for each source and each frequency bands is summarized in Table 3. It ranges from $5.9 \mathrm{mK}$ to $80.9 \mathrm{mK}$, depending on the frequencies and the sources. The line intensity was calibrated by the chopper wheel method. The antenna temperature is divided by the main beam efficiency of 0.5 to obtain the main-beam temperature, $T_{\mathrm{MB}}$. The observation data were first reduced with the ATNF analysis programs, ASAP, and then detailed analyses were carried out using our own codes.

\section{Results and Discussion}

\subsection{Observed Spectra and Line Parameters}

Figures $2 \mathrm{a}$ - 2f show compressed spectra from $85 \mathrm{GHz}$ to $101 \mathrm{GHz}$ toward CO Peak 1, NQC2, N79, N44C, N11B, N113, and N159W, respectively. In preparation of these data, we corrected slight intensity variation among the subscans for 1.5 hours by using the intensity of the strongest line of the observing band. For the compressed spectra, we subtracted the baseline by using the 5th-order polynomial over the $2.2 \mathrm{GHz}$ region. Although the standing waves caused by the telescope system are still visible, we were readily able to identify emission lines, because a typical line width observed toward the target sources (5 $\mathrm{km} \mathrm{s}^{-1}$ ) is narrower than the period of the standing waves. Lines are identified with the aid of the spectral line database CDMS (Müller et al. 2001; Müller et al. 2005). We used the ${ }^{13} \mathrm{CO}$ line velocity $\left(V_{\mathrm{LSR}}\right)$ of $229,266,285,282,232,235$ and $238 \mathrm{~km} \mathrm{~s}^{-1}$ for CO Peak 1, NQC2, N11B, N44C, N79, N113, and N159W, respectively, to identify molecular lines. We detected 8 molecules $\left(\mathrm{CO},{ }^{13} \mathrm{CO}, \mathrm{CS}, \mathrm{SO}, \mathrm{CCH}, \mathrm{HCO}^{+}, \mathrm{HCN}\right.$, and $\mathrm{HNC}$ ) in the seven

sources, $c-\mathrm{C}_{3} \mathrm{H}_{2}$ in N79, N44C, N11B, N113, and N159W, and CN in N79, N44C, and N113 (Table 4). $\mathrm{N}_{2} \mathrm{H}^{+}$is marginally detected in N113 and N159W. The detection criterion is that the line is detected at the expected velocity with the significance of $5 \sigma$ or higher in 
the integrated intensity. Note that the $c-\mathrm{C}_{3} \mathrm{H}_{2}$ line was not covered with the frequency setting for CO Peak 1. Since line intensities in CO Peak 1 and NQC2 are typically weaker by a factor of 2 than the other sources, a fewer lines were detected in these sources. The signal-to-noise ratio is rather poor for N113 and N159W in comparison with the previous studies (Wang et al. 2009; Heikkilä et al. 1999; Chin et al. 1997), and hence, only strong lines were detected in this study.

The spectral profile of each molecular line is shown in Figures 3 and 4 . For Figures 3 and 4 and Table 4 , the baselines are subtracted in a narrow range (typically $100 \mathrm{MHz}$ ) by using the 5th-order polynomials to remove the base line ripples. We conducted Gaussian fitting to each base-line-subtracted spectral line to obtain the $V_{\mathrm{LSR}}, \Delta v$, and the peak $T_{\mathrm{MB}}$, where the observed frequencies are converted to the LSR velocities based on the rest frequency of each transition given in CDMS. Derived line parameters are summarized in Table 4.

Although the seven sources have different star-formation activities, as mentioned in Section 2, the spectral patterns of the seven sources look similar to one another, except for a slight difference discussed later. In order to reveal the similarity, we inspected the molecule-to-molecule correlation of the integrated intensities of the 7 sources. The correlation coefficient $c$ is calculated as

$$
c=\frac{\sum\left(x_{i}-\bar{x}\right)\left(y_{i}-\bar{y}\right)}{\sqrt{\sum\left(x_{i}-\bar{x}\right)^{2} \sum\left(y_{i}-\bar{y}\right)^{2}}}
$$

where $x_{i}$ and $y_{i}$ are observed integrated intensities for the $i$-th source. $\bar{x}$ and $\bar{y}$ are the averages of $x_{i}$ and $y_{i}$, respectively. If the correlation is good, the relative abundance between the two species is similar despite the different column densities along the line of sight. Table 5 shows the results for the 7 species. The correlation coefficients are generally higher than 0.9 , except for the ${ }^{13} \mathrm{CO}$, indicating that the relative abundances of any pair of $\mathrm{CCH}, \mathrm{HCN}, \mathrm{HCO}^{+}, \mathrm{HNC}, \mathrm{CS}$, and $\mathrm{SO}$ are similar among the 7 sources. On the other 
hand, the correlation coefficients between ${ }^{13} \mathrm{CO}$ and the other species are lower than 0.8 . Thus the abundances of $\mathrm{CCH}, \mathrm{HCN}, \mathrm{HCO}^{+}, \mathrm{HNC}, \mathrm{CS}$, and $\mathrm{SO}$ relative to ${ }^{13} \mathrm{CO}$ show more source-to-source variation. This implies that the emmiting region of the ${ }^{13} \mathrm{CO}$ line is different from those of the other species, because of its lower critical density and/or saturation effect.

In this study, we observed the position involving the bright $24 \mu \mathrm{m}$ source for $\mathrm{N} 44 \mathrm{C}$. On the other hand, Chin et al. (1997) observed various molecular lines toward the position offset by $1^{\prime}$ with the SEST $15 \mathrm{~m}$ telescope, whose beam does not involve the bright $24 \mu \mathrm{m}$ source. We confirmed that the spectral pattern constructed from the line intensity data observed by (Chin et al. 1997) is very similar to our result. This fact further suggests that star-formation activities would not seriously affect the chemical compositon at a 10 pc-scale.

Thus, an important result of this study is that the observed spectral pattern with Mopra at $3 \mathrm{~mm}$ is similar for all the observed sources, although these sources have different star formation activities. The previous studies focus on particular sources (e.g. N113 and N159W) with higher sensitivity and often with higher excitation lines. As a result, the chemical composition derived from these studies is more or less specific to each source. In contrast, we are extracting a common feature of the chemical composition averaged over the molecular cloud scale (10 pc scale) with Mopra. Such a chemical composition is almost free from the influence of individual star formation activities, and can be used to study the characteristic chemical composition specific to low-metallicity conditions.

A similar result for molecular-cloud scale chemical compositions in a much larger scale (kpc scale) is recently reported for the spiral arm clouds of the external galaxy M51 (Watanabe et al. 2014). They observed the two positions, P1 and P2, in the spiral arm of M51, which are separated by 20", with the IRAM 30 m telescope. Although the star formation efficiency evaluated from the $\mathrm{H} \alpha$ and $24 \mu \mathrm{m}$ emission is higher in $\mathrm{P} 1$ than in $\mathrm{P} 2$ 
by a factor of 1.5 , the chemical composition is essentially the same in the two positions. Thus, local star formation and its feedback do not strongly affect the spectral pattern observed at a resolution of a molecular-cloud scale.

\subsection{Comparison with Galactic Molecular Cloud}

Comparison of our result in the LMC with the Galactic sources is not easy. Apparently, the observed spectral pattern does not originate from star forming cores, but reflects the average over the molecular cloud scale. In fact, the spectral pattern observed in this study is much different from the Galactic star forming regions such as Orion KL and NGC 2264 (Watanabe et al. 2015) (Figure 2). Hence, we need the chemical composition of the Galactic sources averaged over the molecular cloud scale (10 pc) for fair comparison. However, such data are not available, except for the Galactic center region which has peculiar physical and chemical conditions (e.g. Jones et al. 2012, 2013). Although molecular clouds harbors dense cores and star forming cores, most parts of them would be less dense. Hence, it is worth comparing our result in the LMC with the chemical composition of the translucent clouds as the best possible effort.

Turner and his collaborators observed the $\mathrm{HCO}^{+}, \mathrm{HCN}, \mathrm{HNC}$, CS and SO lines toward many translucent clouds with the total $\mathrm{A}_{\mathrm{v}}$ of about $2-4$ magnitude and $\mathrm{H}_{2}$ density of $10^{3}-10^{4} \mathrm{~cm}^{-3}$ (Turner 1994, 1995a b, 1996; Turner et al. 1997; Turner 1998; Turner et al. 1999, 2000). We averaged the intensities of 38 translucent clouds, and prepared a hypothetical spectrum of a translucent cloud, as shown in Figure 21.

The hypothetical spectrum is compared with the observed spectra in the LMC. The intensities of $\mathrm{HCN}$ and $\mathrm{HNC}$ relative to that of $\mathrm{HCO}^{+}$are apparently brighter in the hypothetical cloud than the spectra in the LMC clouds. This is due to the lower elemental 
abundance of nitrogen in the LMC than in the Galaxy (Table 1), as described in section 4.3. Except for the difference of N-bearing species, the LMC spectra seem to resemble the hypothetical spectrum of the translucent cloud. Note that molecules other than HCN, $\mathrm{HCO}^{+}, \mathrm{HNC}, \mathrm{CS}$, and $\mathrm{SO}$ lines are not included in the hypothetical spectrum due to the lack of the available data for the translucent clouds.

In order to see whether such comparison with the hypothetical translucent cloud is meaningful, we compare the hypothetical translucent cloud spectrum with the spectrum taken toward the spiral arm of M51 (Watanabe et al, 2014), which has a similar metallicity to the solar neighborhood. Since the $\mathrm{H}_{2}$ density derived from the two transitions of $\mathrm{H}_{2} \mathrm{CO}\left(1_{01}-0_{00}\right.$ and $\left.2_{02}-1_{01}\right)$ by the statistical equilibrium calculation under the large-velocity-gradient (LVG) approximation (Goldreich \& Kwan 1974) is $\sim 10^{4} \mathrm{~cm}^{-3}$ in the spiral arm of M51 (Nishimura et al. in prep.), the contribution of less dense parts of molecular clouds is dominant. The hypothetical spectral pattern of the Galactic translucent cloud looks similar to that observed in M51. Hence, it is most likely that the lower HCN and $\mathrm{HNC}$ abundances relative to $\mathrm{HCO}^{+}$in the $\mathrm{LMC}$ clouds originate from the low elemental abundance of nitrogen.

\subsection{Molecular Abundances}

In this section, we evaluate beam-averaged column densities of $\mathrm{HCN}, \mathrm{HCO}^{+}, \mathrm{HNC}, \mathrm{CS}$, and SO. Considering that we are looking molecular cloud scale (10 pc) chemical composition in our observations of the LMC, we assume the $\mathrm{H}_{2}$ density to be $5 \times 10^{3}-5 \times 10^{4} \mathrm{~cm}^{-3}$. In this condition, most of the observed lines except for ${ }^{13} \mathrm{CO}$ are sub-thermally excited, and the level population would naturally be in the non-LTE condition. Hence, it is safe to use the LVG statistical equilibrium calculation to derive the column densities Goldreich \& Kwan 1974). Drawback is that we have to assume the density and the temperature. 
However, these two parameters can be constrained within reasonable ranges. The $\mathrm{H}_{2}$

density is reported to be of the order of $10^{4}-10^{6} \mathrm{~cm}^{-3}$ for N113 and N159W (Wang et al. 2009; Heikkilä et al. 1999). Since these are mostly derived by using the higher excitation lines with high critical density, the emitting regions of the $3 \mathrm{~mm}$ lines observed in this study would originate from less dense regions. Hence, we employed the $\mathrm{H}_{2}$ density range mentioned above. The temperature range is set from $10 \mathrm{~K}$ to $30 \mathrm{~K}$, according to the result of Heikkilä et al. (1999). Under these assumptions, we calculated the column density of each molecule directly from the observed intensity. We basically employed the RADEX code (Schöier et al. 2005) for this purpose except for CCH: we prepared the statistical equilibrium calculation code for $\mathrm{CCH}$ by employing the collisional cross sections reported in RADEX. The results are summarized in Table 6. Although the column densities are sensitive to the assumed $\mathrm{H}_{2}$ density and temperature, the abundance ratios such as $\mathrm{HCN} / \mathrm{HCO}^{+}$are much less sensitive. The abundances relative to $\mathrm{HCO}^{+}$are shown in Table 7 . Note that these results does not vary significantly, if we assume the $\mathrm{H}_{2}$ density of $10^{5} \mathrm{~cm}^{-3}$. We evaluated the error from the range of deviation due to different physical conditions as well as the rms noise of the spectra. For N113 and N159W, the derived abundance relative to $\mathrm{HCO}^{+}$are almost consistent with the values calculated from the previous reports, as shown in Table 7 .

\subsubsection{Nitrogen Bearing Molecules}

One of the characteristic features in the chemical compositions in the LMC is the deficiency of the N-bearing molecules due to the low elemental abundance of nitrogen, which is already evident in the spectral pattern. We first discuss this feature by focusing on the $\mathrm{HCN} / \mathrm{HCO}^{+}$and $\mathrm{HNC} / \mathrm{HCO}^{+}$ratios. The $\mathrm{HCN} / \mathrm{HCO}^{+}$and $\mathrm{HNC} / \mathrm{HCO}^{+}$ratio averaged for the 7 sources are $3.4 \pm 0.6$ and $0.8 \pm 0.2$, respectively. For quantitative comparison, we derived the column densities of $\mathrm{HCO}^{+}, \mathrm{HCN}$, and $\mathrm{HNC}$ for the hypothetical translucent 


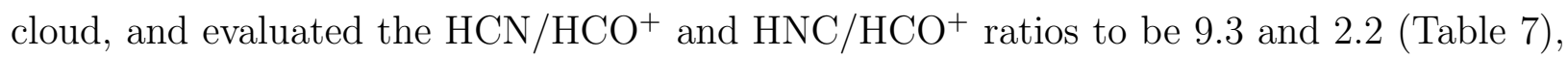
respectively. If the brightest cloud (CB17) among the Galactic translucent clouds in the sample mentioned above is dropped, the ratios are 19.3 and 3.5, respectively. The elemental $\mathrm{N} / \mathrm{O}$ ratio in the LMC is lower by a factor of 3.4 than that in the solar neighborhood (Table 1). The $\mathrm{HCN} / \mathrm{HCO}^{+}$and $\mathrm{HNC} / \mathrm{HCO}^{+}$ratios in the $\mathrm{LMC}$ are also lower by a similar factor than in the Galactic translucent clouds (Table 7). Hence, it is most likely that the deficiency of the N-bearing molecules originates from the elemental deficiency of nitrogen in the LMC.

A similar comparison is also carried out for M51 P1. We evaluated the $\mathrm{HCN} / \mathrm{HCO}^{+}$ and $\mathrm{HNC} / \mathrm{HCO}^{+}$ratios in $\mathrm{M} 51 \mathrm{P} 1$ by using the statistical equilibrium calculations to be 9.6 and 1.7, respectively, for a consistent comparison with the analyses of the LMC (Table 7). These ratios are larger than those in the LMC. This result further suggests that the $\mathrm{HCN} / \mathrm{HCO}^{+}$and $\mathrm{HNC} / \mathrm{HCO}^{+}$ratios are related to the elemental abundance of nitrogen.

Millar \& Herbst (1990) carried out chemical model calculations of dark clouds in the LMC, comparing with chemical compositions of our Galactic molecular clouds. They reported that the abundance of N-bearing species are sensitive to the elemental abundance of N. Specifically, they took account of the lower grain abundance and the higher UV radiation in the LMC than in our Galaxy, and conducted simulations for two types of the elemental abundances; the $\mathrm{N} / \mathrm{O}$ ratios are 0.3 times (model L1) and 0.2 times (model L2) than those in our Galaxy. The resultant abundance ratios are 2.3 and 0.1 for $\mathrm{HCN} / \mathrm{HCO}^{+}$ and 1.3 and 0.09 for $\mathrm{HNC} \mathrm{HCO}^{+}$in their model $\mathrm{L} 1$ and $\mathrm{L} 2$, respectively, at the early time. These are lower than the $\mathrm{HCN} / \mathrm{HCO}^{+}$and $\mathrm{HNC} / \mathrm{HCO}^{+}$ratios calculated for the Galactic conditions, 5.7 and 3.1, respectively. The low abundances of HCN and HNC are thus related to the low elemental abundance of nitrogen.

Next, we focus on the $\mathrm{HNC} / \mathrm{HCN}$ ratio. $\mathrm{HNC}$ is a geometrical isomer of $\mathrm{HCN}$, and 
is less stable by $0.49 \mathrm{eV}$ (Bentley et al. 1993). The $\mathrm{HNC} / \mathrm{HCN}$ ratio reflects production and isomerization mechanisms of these two species in molecular clouds (e.g. Hirota et al. 1998). We evaluated the $\mathrm{HNC} / \mathrm{HCN}$ ratio in the $\mathrm{LMC}$ to be $0.26 \pm 0.08$, which is lower than the ratio in typical dark clouds $(0.54 \sim 4.5$, Hirota et al. (1998)). It is close to the ratio reported for some Galactic diffuse clouds, where the HCN and HNC lines are detected against the continuum sources $(0.21 \pm 0.05$, Liszt \& Lucas (2001)). Hirota et al. (1998) reported that the $\mathrm{HNC} / \mathrm{HCN}$ ratio is lower under higher-temperature environments: the ratio decreases above $24 \mathrm{~K}$. The relatively low ratios observed in the LMC clouds may originate from warmer temperature conditions than in the Galactic translucent clouds due to higher UV field and/or lower grain abundance in the LMC. Wang et al. (2009) pointed out that the lower ratio of $\mathrm{HNC} / \mathrm{HCN}$ in N113 reflects the effect of strong UV radiation, on the basis of the observation of $8 \mu \mathrm{m}$ emmision of PAH (Wong et al. 2006). In this study, we found the $\mathrm{HNC} / \mathrm{HCN}$ ratio is lower even in the quiescent clouds (Category A and B). Our result suggests that the warmer temperature caused by the lower abundance of dust grains in the low metallicity conditions would be responsible for the lower $\mathrm{HNC} / \mathrm{HCN}$ ratio (Section 4.3.3).

Finally, we briefly discuss non-detection of $\mathrm{N}_{2} \mathrm{H}^{+}$except for $\mathrm{N} 113$ and N159W in this study. This seems to originate mainly from the low elemental abundance of nitrogen. In addition, the warm condition would contribute to diminishing the $\mathrm{N}_{2} \mathrm{H}^{+}$abundance. $\mathrm{N}_{2} \mathrm{H}^{+}$ is easily destroyed by the proton-transfer reaction with CO (Bergin et al. 2002; Jørgensen 2004). Since $\mathrm{CO}$ is not well depleted in warm peripheries $(T>20 \mathrm{~K})$, the $\mathrm{N}_{2} \mathrm{H}^{+}$abundance would further decrease by this mechanism. 


\subsection{2. $\mathrm{CCH}$}

Another characteristic feature is a relatively high abundance of $\mathrm{CCH}$ in comparison with the Galactic translucent clouds. We calculated the abundance ratio of $\mathrm{CCH} / \mathrm{HCO}^{+}$ for the seven observed clouds in LMC, and also for three translucent cloud (CB17, CB24, CB228) observed by Turner, under the assumption of the $\mathrm{H}_{2}$ density of $5 \times 10^{3}-5 \times 10^{4}$ $\mathrm{cm}^{-3}$ and the gas kinetic temperature of $10-30 \mathrm{~K}$, as shown in Table 7. The ratio of $\mathrm{CCH} / \mathrm{HCO}^{+}$is higher in the LMC $(\sim 12)$ than in the Galactic translucent clouds $(\sim 4.2)$ by a factor of 3 . Note the elemental $\mathrm{C} / \mathrm{O}$ ratio is lower in the LMC ( 0.33) than in the solar neighborhood of our Galaxy $(\sim 0.60)$ by a factor of 0.55 . If this difference of the $\mathrm{C} / \mathrm{O}$ ratio is taken into account, the $\mathrm{CCH} / \mathrm{HCO}^{+}$ratio in the $\mathrm{LMC}$ is higher than the ratio expected from the elemental $\mathrm{C} / \mathrm{O}$ ratio by factor of 5 .

It is generally thought that $\mathrm{CCH}$ is abundant in the photodissociation region (PDR) illuminated by UV radiation (e.g. Martín et al. 2014). Since the metallicity in the LMC is lower than our Galaxy, the abundance of dust grains is accordingly lower. Hence, the extinction of the UV radiation by dust grains is less effective for a given column density of $\mathrm{H}_{2}$. For this reason, the PDR tends to be extended deeper into the clouds in the LMC, which would be responsible to the relatively high abundance of $\mathrm{CCH}$. In the PDR, growth of large carbon-chain molecules are suppressed by competitive photodissociation process. In fact, the abundance ratio of $c-\mathrm{C}_{3} \mathrm{H}_{2}$ relative to $\mathrm{CCH}$ is lower in the LMC than in the solar neighborhood of our Galaxy; we calculated the abundance ratio of $\mathrm{CCH} / c-\mathrm{C}_{3} \mathrm{H}_{2}$ to be $\sim 10$ and $\sim 3$ in the LMC and the Galactic translucent clouds, respectively. 


\subsection{3. $\mathrm{CH}_{3} \mathrm{OH}$}

It is worth noting non-detection of $\mathrm{CH}_{3} \mathrm{OH}$ in our survey. We obtained an upper limit of $\mathrm{CH}_{3} \mathrm{OH}$ intensity and column density in the seven clouds in the LMC. The abundance ratio of $\mathrm{CH}_{3} \mathrm{OH} / \mathrm{HCO}^{+}$in the $\mathrm{LMC}$ are comparable to or lower than in the Galactic translucent clouds. This result can also be interpreted in terms of a stronger UV effect. $\mathrm{CH}_{3} \mathrm{OH}$ is thought to be produced by hydrogenation of $\mathrm{CO}$ on dust grains, and is liberated into the gas phase by thermal and/or non-thermal desorption (e.g. Watanabe \& Kouchi 2002). Since the adsorption temperature of CO is $20 \mathrm{~K}$, CO is not well depleted onto dust grains in the warm condition, where the UV heating is effective. Furthermore, an evaporation time of the $\mathrm{H}$ atom rapidly becomes shorter for higher temperature, giving a less chance to react with $\mathrm{CO}$. Hence, $\mathrm{CH}_{3} \mathrm{OH}$ is not formed efficiently in warm conditions as those present in the LMC. This is also suggested by Shimonishi et al. (2015) on the basis of the infrared observation of solid $\mathrm{CO}_{2}$. The dust temperature of the LMC is generally higher

than that of our Galay, according to the infrared observations Aguirre et al. 2003; Sakon et al. 2006), which is supposed to be caused by the strong UV radiation field (Bel et al. 1986). However, the present upper limits of $\mathrm{CH}_{3} \mathrm{OH} / \mathrm{HCO}^{+}$are not stringent enough to conclude this effect. Further sensitive observations are necessary to confirm the deficiency of $\mathrm{CH}_{3} \mathrm{OH}$ in the LMC.

\subsection{Abundant S-bearing Molecules in CO Peak 1}

Although the spectra observed toward the seven sources in the LMC are similar to one another as a whole, we find a slight but significant difference of the CS and SO abundance in CO Peak 1. Figure 5 shows correlation diagrams of integrated intensities for the five species $\left(\mathrm{HCO}^{+}, \mathrm{HCN}, \mathrm{HNC}, \mathrm{CS}\right.$, and SO) for the three sources (N113, N44C, and CO Peak 1). The sulfur-bearing species (CS and SO) tend to be abundant in CO Peak 1. To confirm 
this trend, we evaluated the $\mathrm{CS} / \mathrm{HCO}^{+}$and $\mathrm{SO} / \mathrm{HCO}^{+}$abundance ratios for the seven LMC clouds from the column densities. The results are shown in Figure 6. The abundances of $\mathrm{CS}$ and $\mathrm{SO}$ relative to $\mathrm{HCO}^{+}$tend to be higher in $\mathrm{CO}$ Peak 1 by factor of 2 than the averages for the other 6 sources. Star-formation activities would enhance the abundance of the sulfur-bearing molecules, because it is generally thought that liberation of sulfur atoms and/or sulfur-bearing molecules by outflow shocks and photostellar heating is responsible for the enhancement (e.g. Wakelam et al. 2005, 2011). However, the slight enhancement of $\mathrm{CS}$ and $\mathrm{SO}$ relative to $\mathrm{HCO}^{+}$in $\mathrm{CO}$ Peak 1 is not an effect of star-formation activity, because CO Peak 1 does not harbor high-mass YSOs. In our observations of the seven sources, the abundances of sulfur-bearing species are the highest in the quiescent cloud, CO Peak 1. We here consider the two possible reasons for this result.

First, the trend may be caused by depletion of S in evolved molecular clouds, or by deficiency of $\mathrm{HCO}^{+}$in young molecular clouds. In molecular clouds, sulfur is believed to be heavily depleted on dust grains. In chemical models, the gas-phase abundance of sulfur is usually assumed to be only $1 / 100$ of the total elemental abundance (e.g. Wakelam et al. 2011; Aikawa et al. 2008; Leung et al. 1984; Graedel et al. 1982). On the other hand, it is observationally known that sulfur is much less depleted in diffuse clouds than in molecular clouds (Lehner et al. 2004; Van Steenberg \& Shull 1988; Morton 1974). Hence, sulfur would be depleted onto dust grains along molecular-cloud formation. The higher $\mathrm{CS} / \mathrm{HCO}^{+}$and $\mathrm{SO} / \mathrm{HCO}^{+}$ratio in $\mathrm{CO}$ Peak 1 than in more evolved clouds suggests that sulfur depletion is not yet completed in CO Peak 1. Because of the low metallicity, extinction of UV and visible light is less effective in the LMC than in our Galaxy for a given $\mathrm{H}_{2}$ column density, and the dust temperature of the molecular clouds (in particular the temperature of their peripheries) is expected to be higher in the LMC than in our Galaxy (Aguirre et al. 2003, Sakon et al. 2006). The adsorption temperature of sulfur is estimated to be about 20 $\mathrm{K}$ by using the adsorption energy of $1100 \mathrm{~K}$ (Ruffle \& Herbst 2000). Hence, the sulfur 
depletion would be less efficient for molecular clouds in the LMC. As a result, sulfur-bearing molecules would still be abundant in the gas phase. The decrease of the $\mathrm{CS} / \mathrm{HCO}^{+}$and $\mathrm{SO} / \mathrm{HCO}^{+}$ratios may reflect gradual depletion of sulfur along the evolution of molecular clouds. However, it should be noted that such enhancement of sulfur-bearing molecules is not apparently seen in another quiescent cloud, NQC2. Hence, this trend may be specific to CO Peak 1, or NQC2 may be more evolved than CO Peak 1.

Secondly, the above results is due to the low abundance of $\mathrm{HCO}^{+}$in $\mathrm{CO}$ Peak 1 . If the average density is relatively lower in CO Peak 1 than the other sources, the ionization degree is expected to be higher in CO Peak 1 due to contribution of photoionization. Since $\mathrm{HCO}^{+}$is produced by the reaction of $\mathrm{H}_{3}{ }^{+}+\mathrm{CO}$, its formation rate depends on the $\mathrm{H}_{3}{ }^{+}$ ion abundance. The enhancement of ionization degree (i.e. the electron abundance) by the photoionization leads to a lower abundance of $\mathrm{H}_{3}{ }^{+}$, and thereby a lower abundance of $\mathrm{HCO}^{+}$. Above all, the $\mathrm{HCO}^{+}$abundance may be lower in $\mathrm{CO}$ Peak 1, which would also contributes to the trend observed in Figure 6.

Relative contributions of the above two possibilities are difficult to be discriminated only from the present data. It is worth noting that the $\mathrm{CS} / \mathrm{HCN}$ ratio seems to show a similar trend to the $\mathrm{CS} / \mathrm{HCO}^{+}$ratio in spite of large uncertainties (Figure 7). This suggests that the first possibility may work at least partly. Although further statistical studies are necessary for a definitive conclusion, the $\mathrm{CS} / \mathrm{HCO}^{+}$and $\mathrm{SO} / \mathrm{HCO}^{+}$ratios may be used as an evolutionary tracer of molecular clouds.

\section{Summary}

In this paper, we have revealed the chemical composition averaged over the molecularcloud scale on the basis of the spectral line surveys in the 3-mm band toward 7 molecular 
clouds in the LMC. The results are summarized as follows:

1. Eight molecular species, $\mathrm{CCH}, \mathrm{HCN}, \mathrm{HCO}^{+}, \mathrm{HNC}, \mathrm{CS}, \mathrm{SO},{ }^{13} \mathrm{CO}$, and ${ }^{12} \mathrm{CO}$ were identified in the 7 sources. Some other species $\left(c-\mathrm{C}_{3} \mathrm{H}_{2}, \mathrm{~N}_{2} \mathrm{H}^{+}, \mathrm{CN}\right)$ were also detected in several sources.

2. Although CO Peak 1, NQC2, N79, N44C, N11B, N113, and N159W have different star formation activities, their spectral patterns are found to resemble one another. The chemical compositions averaged over a whole moleuclar-cloud scale $(\sim 10 \mathrm{pc}$ scale) are scarcely affected by local star-formation activities.

3. In comparison with the hypothetical spectrum of a translucent cloud in our Galaxy prepared from the literature data, the intensities of $\mathrm{HCN}$ and $\mathrm{HNC}$, relative to the $\mathrm{HCO}^{+}$, are much weaker in the three LMC cloud. This seems to originate from the low elemental abundance of nitrogen in the LMC.

4. In the 7 sources of the $\mathrm{LMC}, \mathrm{CCH}$ is found to be abundant and $\mathrm{CH}_{3} \mathrm{OH}$ is deficient in comparison with our Galaxy. These are interpreted as effects of the strong UV radiation, caused by the lower abundance of dust grains in the low metallicity condition.

5. We find a slight but significant difference in abundance of the sulfur-bearing species relative to $\mathrm{HCO}^{+}$among the seven sources. The abundance ratios of sulfur-bearing species are highest in the starless cloud (CO Peak 1). The decrease of the ratio toward star-forming molecular clouds may reflect the evolution of molecular clouds.

We are grateful to the anonymous reviewer for valuable comments and suggestions. We thank the staff of the Mopra telescope for excellent support. This study is supported 
by Grants-in-Aid from Ministry of Education, Sports, Science, and Technologies of Japan (21224002, 25400223, and 25108005). YN is supported by Grant-in-Aid for JSPS Fellows $(268280)$. 


\section{REFERENCES}

Aguirre, J. E., Bezaire, J. J., Cheng, E. S., et al. 2003, ApJ, 596, 273

Aikawa, Y., Wakelam, V., Garrod, R. T., \& Herbst, E. 2008, ApJ, 674, 993

Anders, E., \& Grevesse, N. 1989, Geochimica et Cosmochimica acta, 53, 197

Barbá, R. H., Rubio, M., Roth, M. R., \& García, J. 2003, AJ, 125, 1940

Bel, N., Viala, Y. P., \& Guidi, I. 1986, A\&A, 160, 301

Bentley, J. A., Huang, C.-M., \& Wyatt, R. E. 1993, The Journal of chemical physics, 98, 5207

Bergin, E. A., Alves, J., Huard, T., \& Lada, C. J. 2002, ApJ, 570, L101

Bica, E., Claria, J., \& Dottori, H. 1992, AJ, 103, 1859

Bresolin, F., Garnett, D. R., \& Kennicutt, Jr., R. C. 2004, ApJ, 615, 228

Chin, Y.-N., Henkel, C., Whiteoak, J. B., et al. 1997, A\&A, 317, 548

Dufour, R., Shields, G., \& Talbot Jr, R. 1982, ApJ, 252, 461

Ellingsen, S. P., Breen, S. L., Caswell, J. L., Quinn, L. J., \& Fuller, G. A. 2010, MNRAS, 404,779

Fukui, Y., \& Kawamura, A. 2010, Annu. Rev. Astron. Astrophys., 48, 547

Garnett, D. R., Edmunds, M. G., Henry, R. B. C., Pagel, B. E. J., \& Skillman, E. D. 2004, AJ, 128, 2772

Goldreich, P., \& Kwan, J. 1974, ApJ, 189, 441

Graedel, T., Langer, W., \& Frerking, M. 1982, ApJS, 48, 321 
Gruendl, R. A., \& Chu, Y.-H. 2009, ApJS, 184, 172

Heikkilä, A., Johansson, L., \& Olofsson, H. 1999, A\&A, 344, 817

Henize, K. G. 1956, ApJS, 2, 315

Hirota, T., Yamamoto, S., Mikami, H., \& Ohishi, M. 1998, ApJ, 503, 717

Johansson, L. E. B., Olofsson, H., Hjalmarson, A., Gredel, R., \& Black, J. H. 1994, A\&A, 291,89

Jones, P. A., Burton, M. G., Cunningham, M. R., Tothill, N. F. H., \& Walsh, A. J. 2013, MNRAS, 433, 221

Jones, P. A., Burton, M. G., Cunningham, M. R., et al. 2012, MNRAS, 419, 2961

Jørgensen, J. K. 2004, A\&A, 424, 589

Kato, D., Ita, Y., Onaka, T., et al. 2012, AJ, 144, 179

Lebouteiller, V., Cormier, D., Madden, S. C., et al. 2012, A\&A, 548, A91

Lehner, N., Wakker, B., \& Savage, B. 2004, ApJ, 615, 767

Leung, C. M., Herbst, E., \& Huebner, W. 1984, ApJS, 56, 231

Liszt, H., \& Lucas, R. 2001, A\&A, 370, 576

Lucke, P., \& Hodge, P. 1970, AJ, 75, 171

Martín, S., Verdes-Montenegro, L., Aladro, R., et al. 2014, A\&A, 563, L6

Meixner, M., Gordon, K. D., Indebetouw, R., et al. 2006, The Astronomical Journal, 132, 2268

Millar, T. J., \& Herbst, E. 1990, MNRAS, 242, 92 
Mizuno, Y., Kawamura, A., Onishi, T., et al. 2010, PASJ, 62, 51

Morton, D. 1974, ApJ, 193, L35

Müller, H. S., Schlöder, F., Stutzki, J., \& Winnewisser, G. 2005, Journal of Molecular Structure, 742, 215

Müller, H. S. P., Thorwirth, S., Roth, D. A., \& Winnewisser, G. 2001, A\&A, 370, L49

Nishimura, Y., Shimonishi, T., Watanabe, Y., et al. in prep.

Ott, J., Henkel, C., Staveley-Smith, L., \& Weiß, A. 2010, ApJ, 710, 105

Paron, S., Ortega, M., Cunningham, M., et al. 2014, A\&A, 572, A56

Pietrzyński, G., Graczyk, D., Gieren, W., et al. 2013, Nature, 495, 76

Ruffle, D. P., \& Herbst, E. 2000, MNRAS, 319, 837

Sakon, I., Onaka, T., Kaneda, H., et al. 2006, ApJ, 651, 174

Schöier, F. L., van der Tak, F. F. S., van Dishoeck, E. F., \& Black, J. H. 2005, A\&A, 432, 369

Seale, J. P., Looney, L. W., Chu, Y.-H., et al. 2009, ApJ, 699, 150

Shimonishi, T., Dartois, E., Onaka, T., \& Boulanger, F. 2015, in press

Shimonishi, T., Nishimura, Y., Watanabe, Y., et al. in prep.

Shimonishi, T., Onaka, T., Kato, D., et al. 2010, A\&A, 514, 12

—. 2013, AJ, 145, 32

Turner, B. 1994, ApJ, 420, 661 
-. 1995a, ApJ, 455, 556

-. 1995b, ApJ, 449, 635

-. 1996, ApJ, 461, 246

-. 1998, ApJ, 501, 731

Turner, B., Herbst, E., \& Terzieva, R. 2000, ApJS, 126, 427

Turner, B., Pirogov, L., \& Minh, Y. 1997, ApJ, 483, 235

Turner, B., Terzieva, R., \& Herbst, E. 1999, ApJ, 518, 699

Van Steenberg, M. E., \& Shull, J. M. 1988, ApJ, 330, 942

Wakelam, V., Ceccarelli, C., Castets, A., et al. 2005, A\&A, 437, 149

Wakelam, V., Hersant, F., \& Herpin, F. 2011, A\&A, 529, 112

Wang, M., Chin, Y.-N., Henkel, C., Whiteoak, J., \& Cunningham, M. 2009, ApJ, 690, 580

Watanabe, N., \& Kouchi, A. 2002, ApJ, 571, L173

Watanabe, Y., Sakai, N., López-Sepulcre, A., et al. 2015, ApJ, in press

Watanabe, Y., Sakai, N., Sorai, K., \& Yamamoto, S. 2014, ApJ, 788, 4

Westerlund, B. E. 1997, The Magellanic Clouds No. 29 (Cambridge University Press)

Whitney, B. A., Sewilo, M., Indebetouw, R., et al. 2008, AJ, 136, 18

Wong, T., Whiteoak, J. B., Ott, J., Chin, Y.-n., \& Cunningham, M. R. 2006, ApJ, 649, 224

Wong, T., Hughes, A., Ott, J., et al. 2011, ApJS, 197, 16 

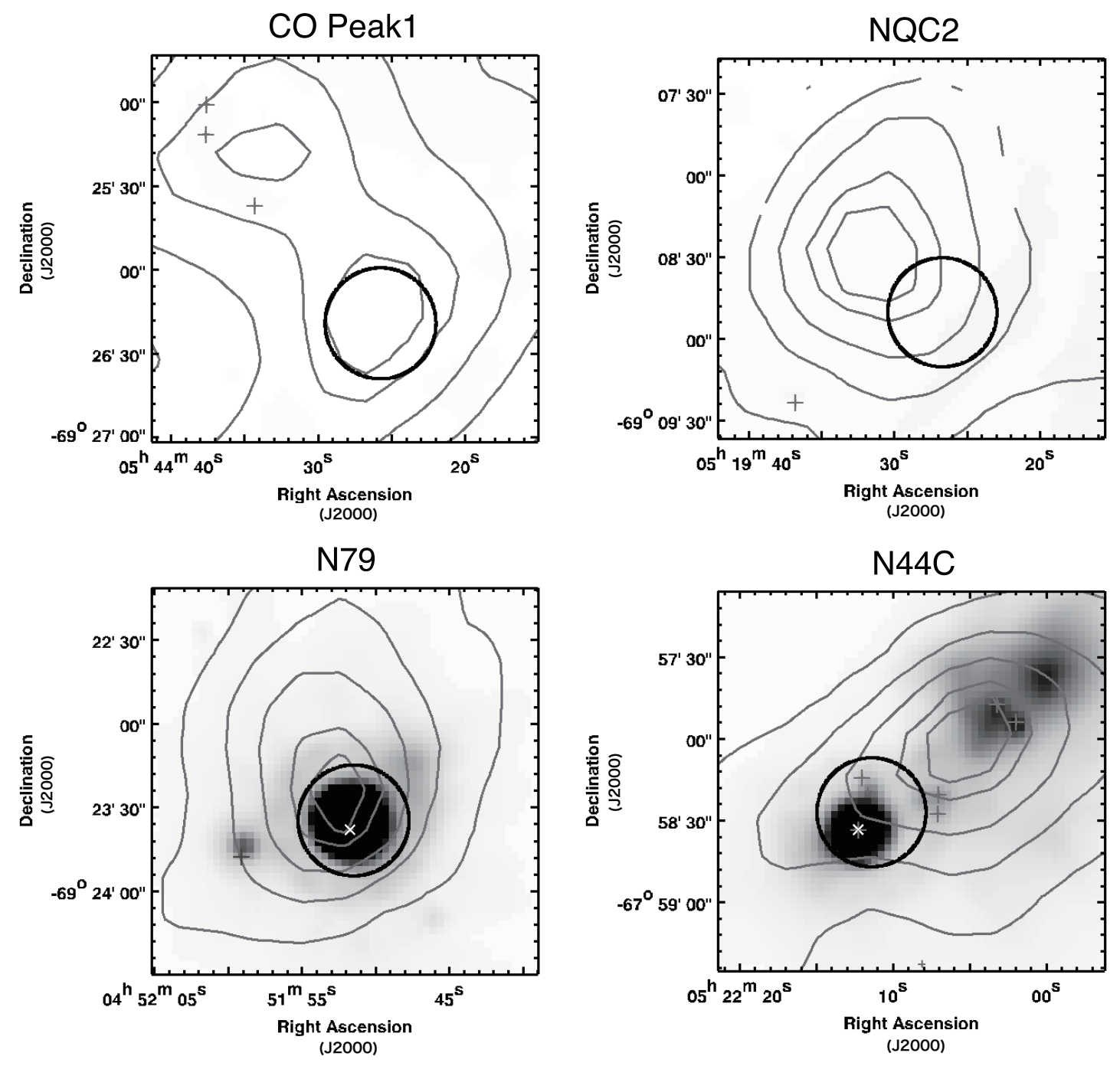

Fig. 1.- $\mathrm{CO}(J=1-0)$ contour (MAGMA) and $24 \mu \mathrm{m}$ image (Spitzer MIPS) of our target molecular clouds. The solid circle represents the beam size of the Mopra telescope in $3 \mathrm{~mm}\left(38^{\prime \prime} \sim 10 \mathrm{pc}\right.$ at the LMC) centered at the observed position. The grey crosses indicate the positions of YSOs candidates reported in Gruendl \& Chu (2009), while the white $\mathrm{x}$-marks indicate the positions of particularly bright high-mass YSOs within or near the beam position. 

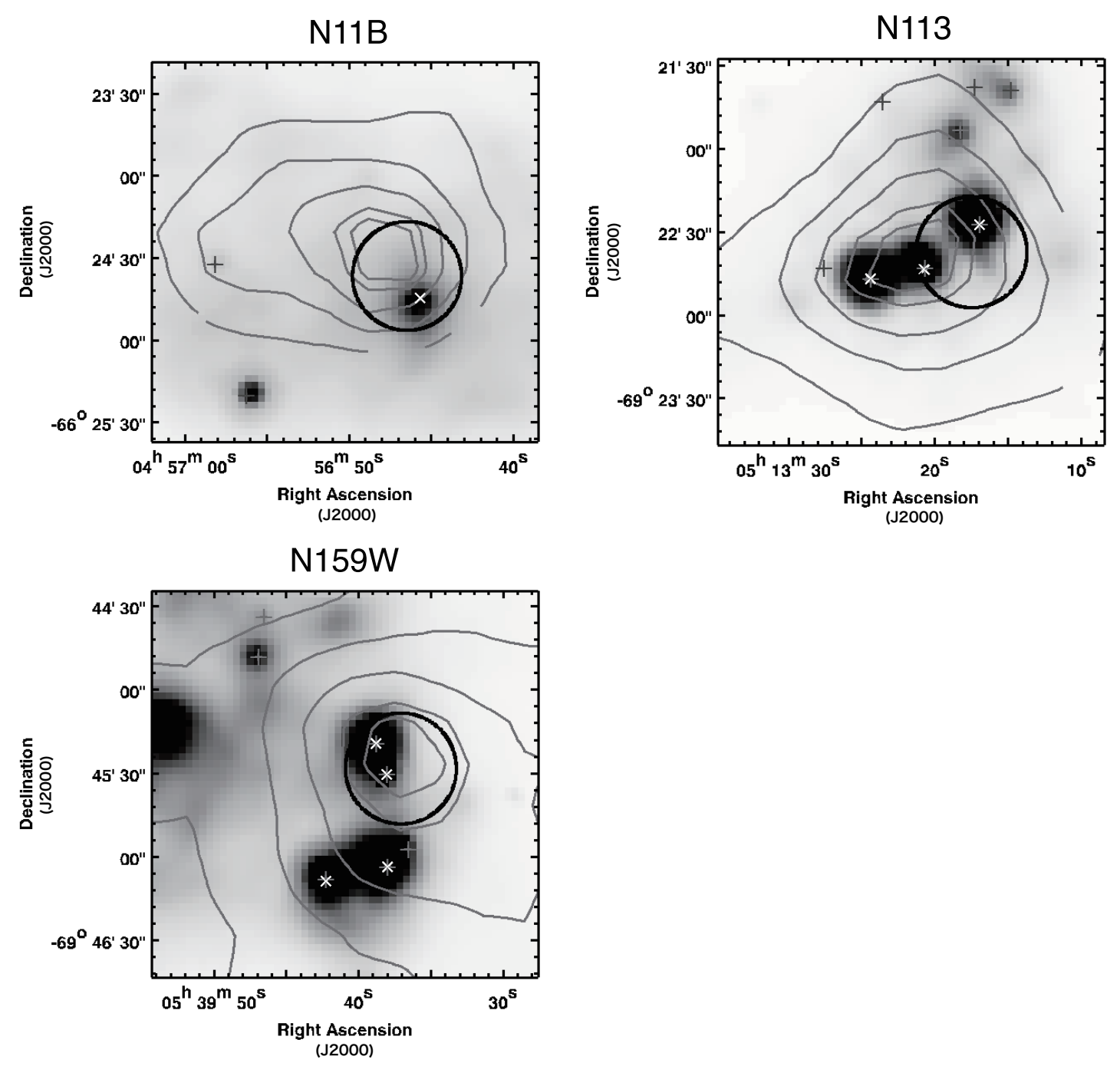

Fig. 1.- Continued 


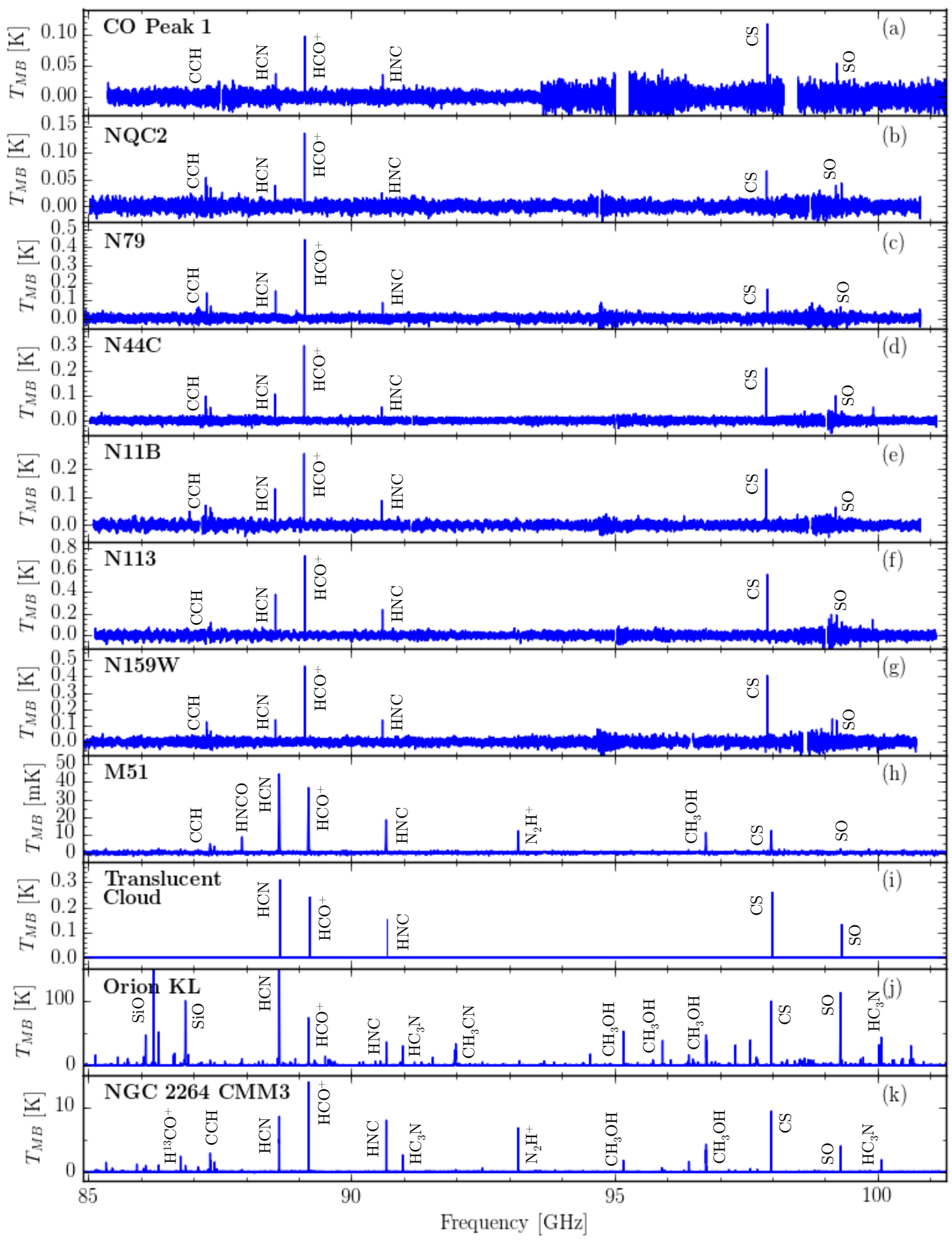

Fig. 2.- Compressed spectra observed toward CO Peak 1 (a), NQC2 (b), N79 (c), N44C (d), N11B (e), N113 (f), N159W (g). The spectrum of the spiral arm of M51 is taken from Watanabe et al. (2014) (h). The composite spectrum of a translucent cloud is prepared by averaging the intensities of $\mathrm{HCN}, \mathrm{HCO}^{+}, \mathrm{HNC}, \mathrm{CS}$ and $\mathrm{SO}$ observed toward 38 translucent clouds by Turner (1995a b, 1996); Turner et al. (1997) (i). The spectrum of Orion KL (j) 

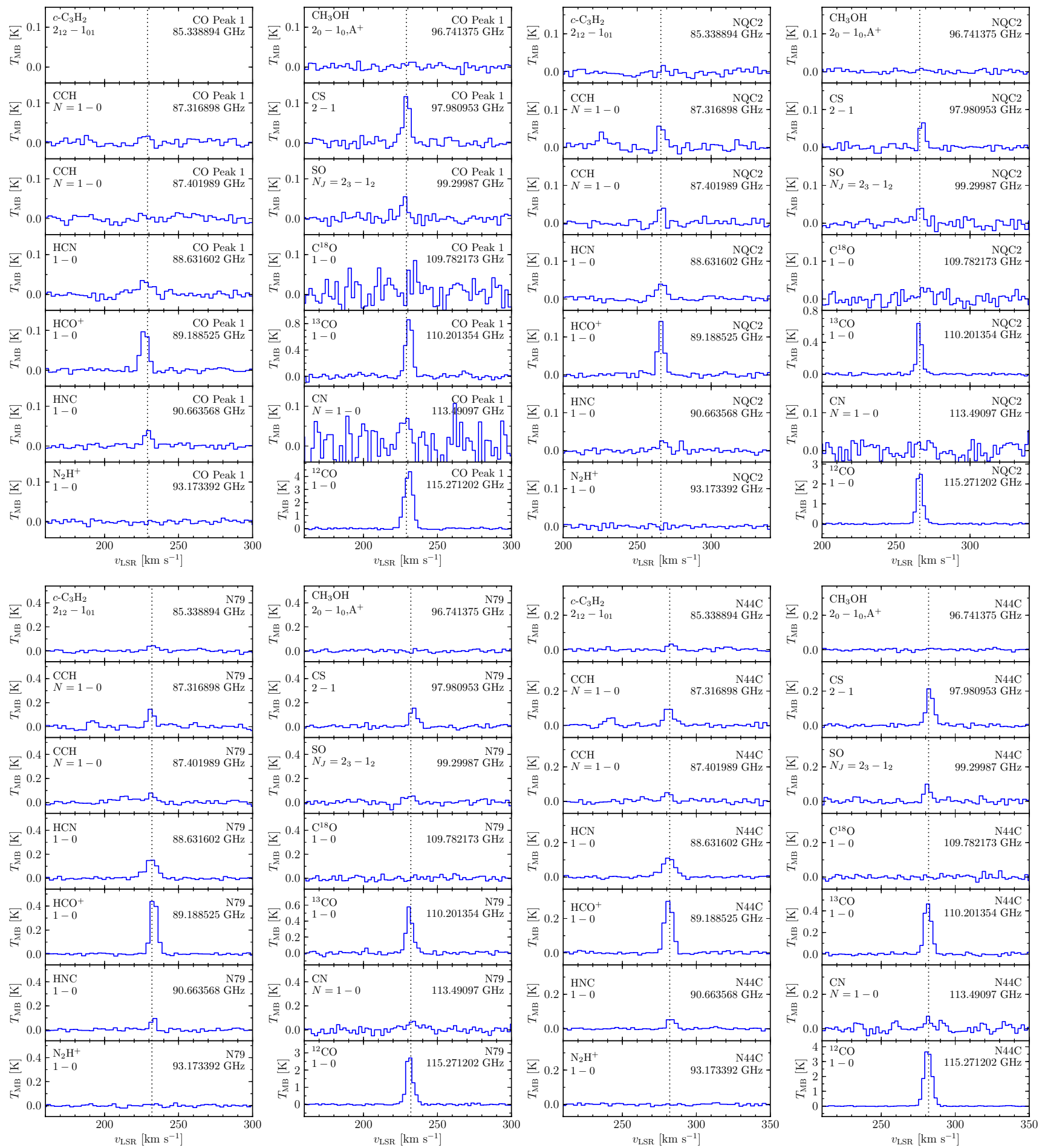

Fig. 3.- Spectral line profiles of individual molecular transitions observed in CO Peak 1,

NQC2, N79, and N44C. 

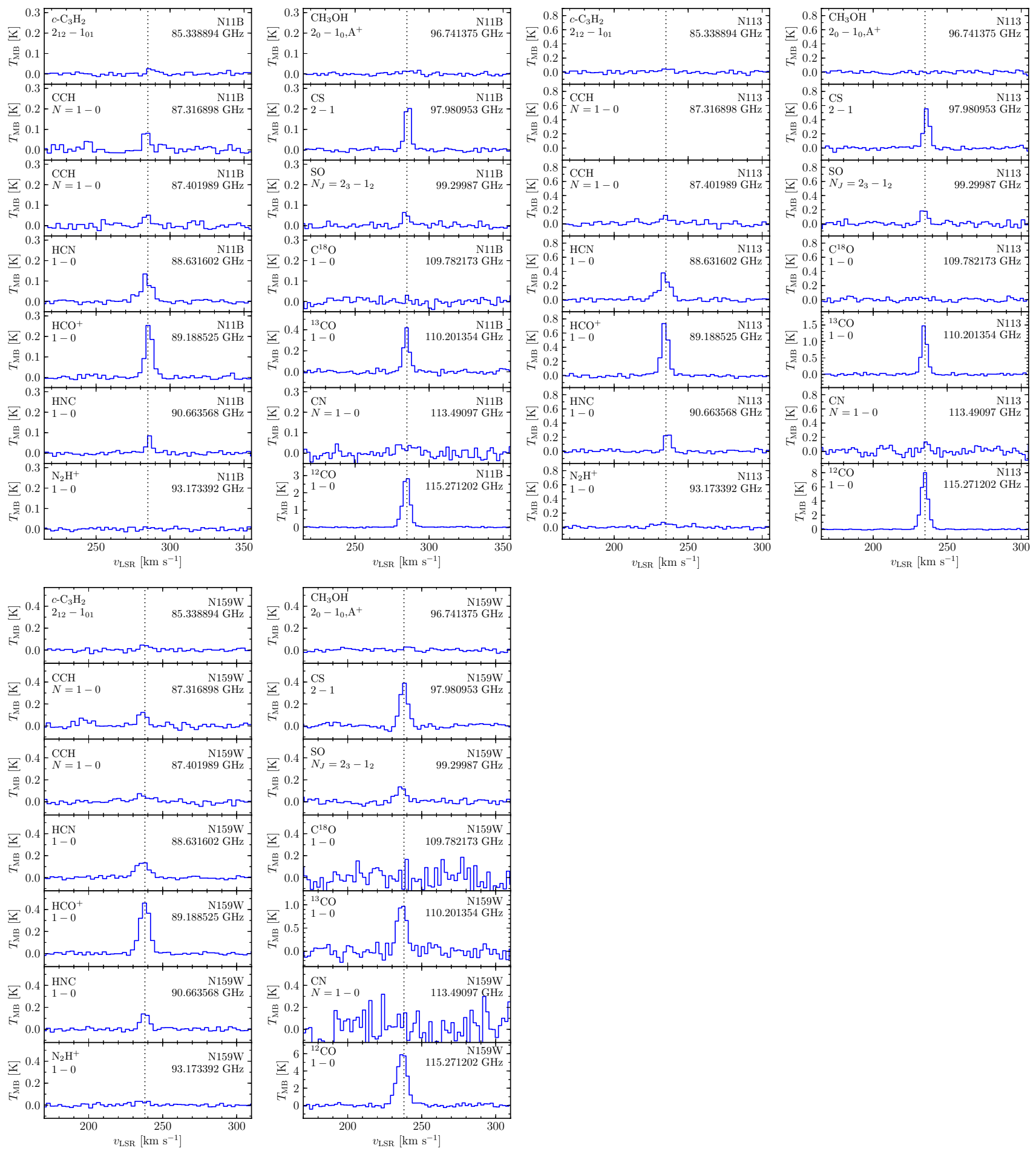

Fig. 4.- Spectral line profiles of individual molecular transitions observed in N11B, N113, and N159W. 

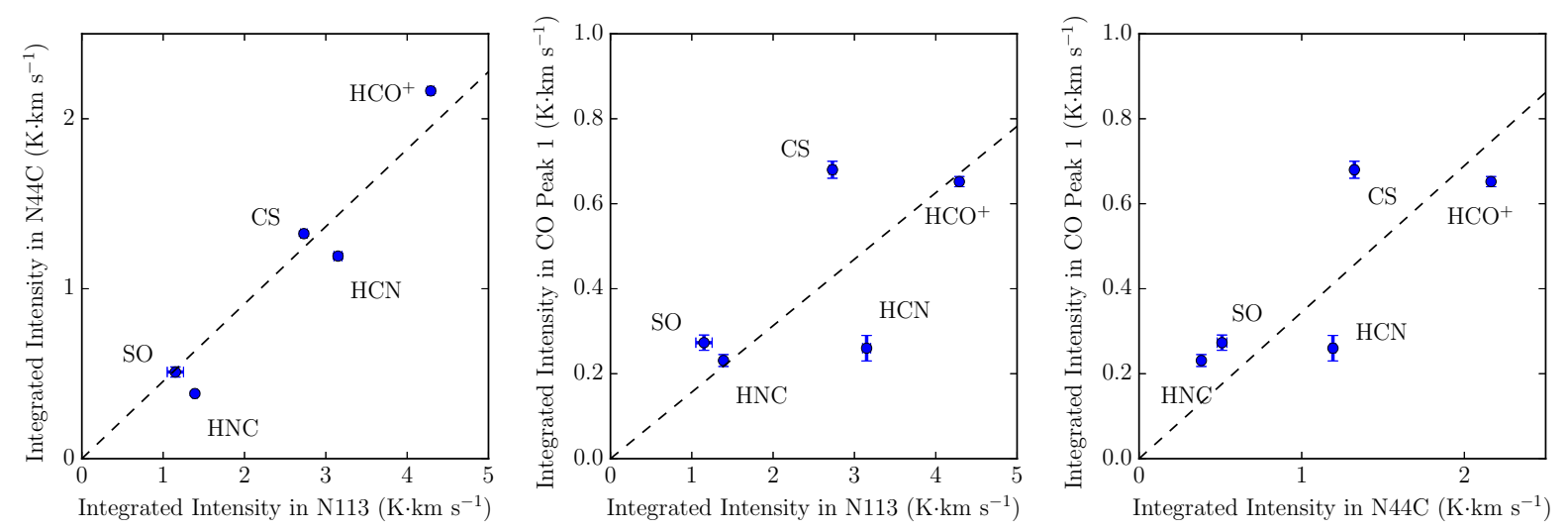

Fig. 5.- Correlation diagrams of integrated intensities of detected species between N113 and N44C (left), N113 and CO Peak 1 (middle), N44C and CO Peak 1(right). The dashed line indicates the average ratio of the integrated intensities of the two sources. The CS and SO lines tend to be relatively stronger in CO Peak 1.

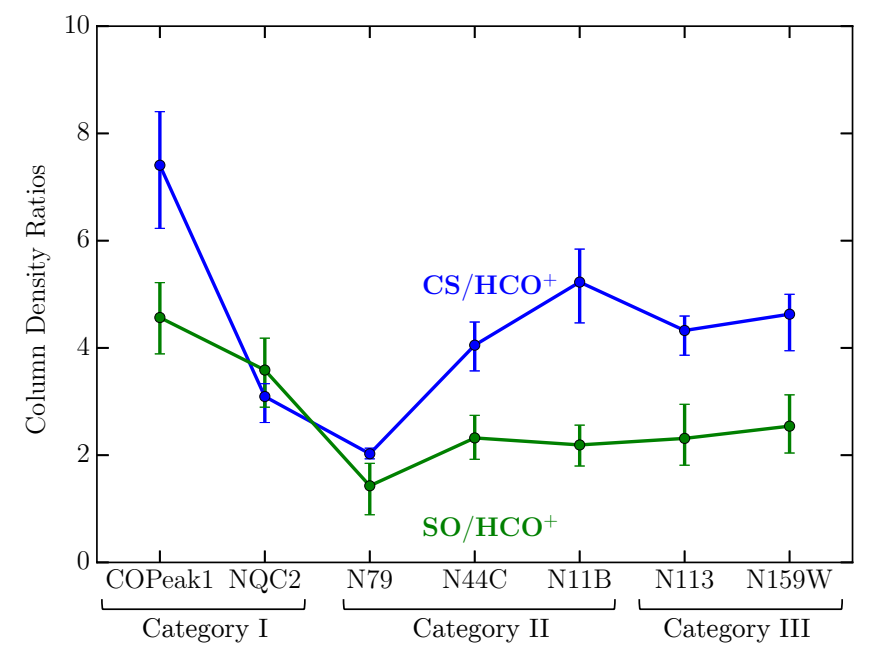

Fig. 6.- Column density ratios of $\mathrm{CS} / \mathrm{HCO}^{+}$and $\mathrm{SO} / \mathrm{HCO}^{+}$in the observed sources. The error bars includes the errors caused by the assumed range of the gas kinetic temperature $(10-30 \mathrm{~K})$ and the gas density $\left(5 \times 10^{3}-5 \times 10^{4} \mathrm{~cm}^{-3}\right)$. The $\mathrm{CS} / \mathrm{HCO}^{+}$and $\mathrm{SO} / \mathrm{HCO}^{+}$ ratio higher in CO Peak 1 (See Section 4.4 for details). 


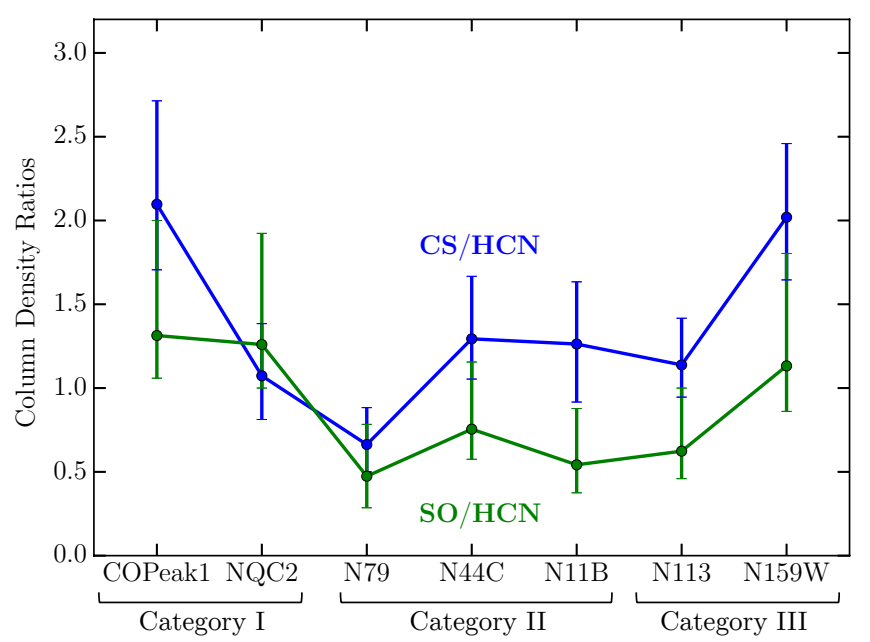

Fig. 7.- Column density ratios of $\mathrm{CS} / \mathrm{HCN}$ and $\mathrm{SO} / \mathrm{HCN}$ in the observed sources. The error bars includes the errors caused by the assumed range of the gas kinetic temperature $(10-30 \mathrm{~K})$ and the gas density $\left(5 \times 10^{3}-5 \times 10^{4} \mathrm{~cm}^{-3}\right)$. A similar trend for CO Peak 1 seen in Figure 6 is also seen.

Table 1. The Elemental Abundances Relative to Hydrogen Nuclei.

\begin{tabular}{lccccc}
\hline \hline \multicolumn{1}{c}{ Galaxy } & $\mathrm{O} / \mathrm{H} \times 10^{4}$ & $\mathrm{C} / \mathrm{H} \times 10^{4}$ & $\mathrm{~N} / \mathrm{H} \times 10^{5}$ & $\mathrm{~S} / \mathrm{H} \times 10^{5}$ & References \\
\hline Milky Way (Solar) & 7.41 & 4.47 & 9.12 & 1.70 & $\mathrm{a}$ \\
Milky Way (Solar) & 8.53 & 3.62 & 11.22 & 1.85 & $\mathrm{a}$ \\
M51 & 6.31 & 3.98 & 15.85 & 1.59 & $\mathrm{~b}, \mathrm{c}$ \\
LMC & 2.40 & 0.79 & 0.87 & 1.02 & $\mathrm{a}$ \\
\hline
\end{tabular}

Note. - a) Dufour et al. (1982, a') Anders \& Grevesse (1989), b) Bresolin et al. (2004), c) Garnett et al. (2004). (The ratio of M51 is the highest one of H II regions in M51.) 


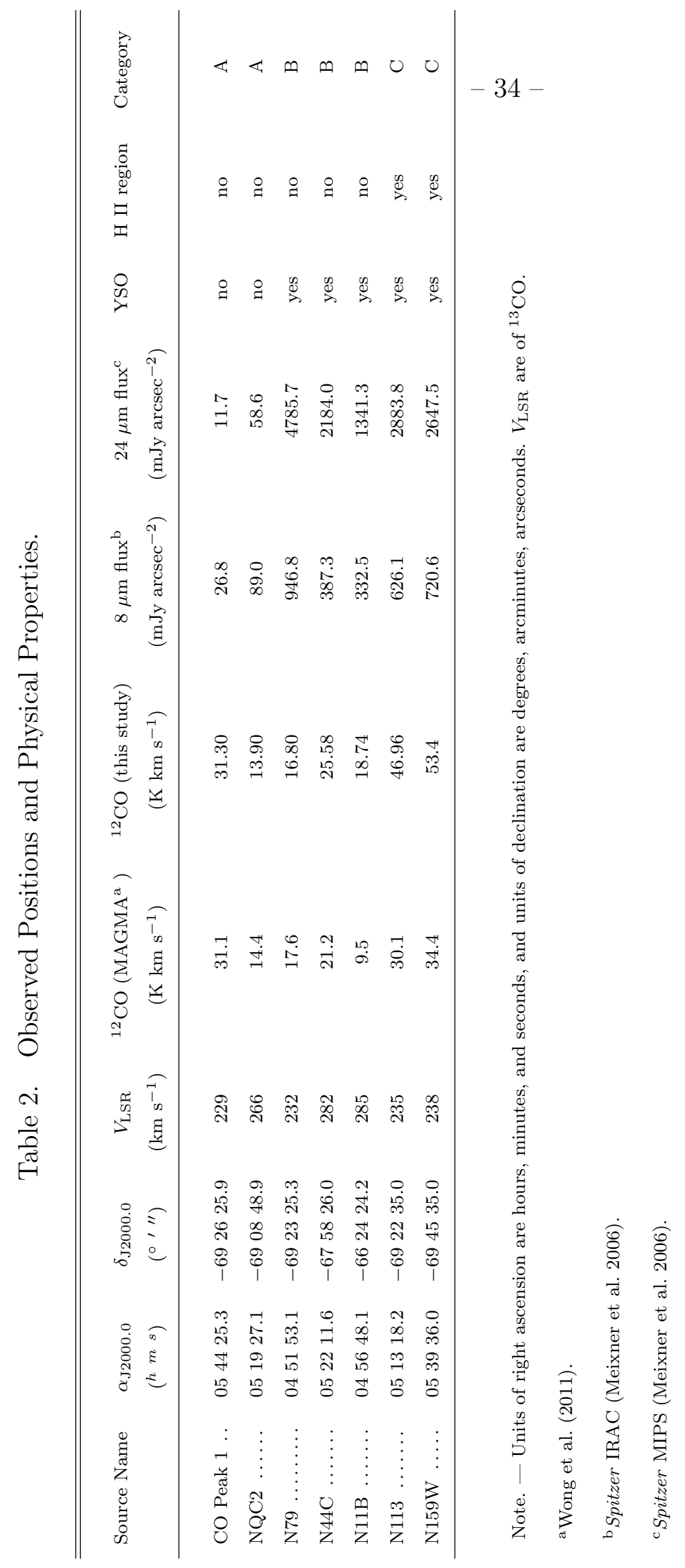




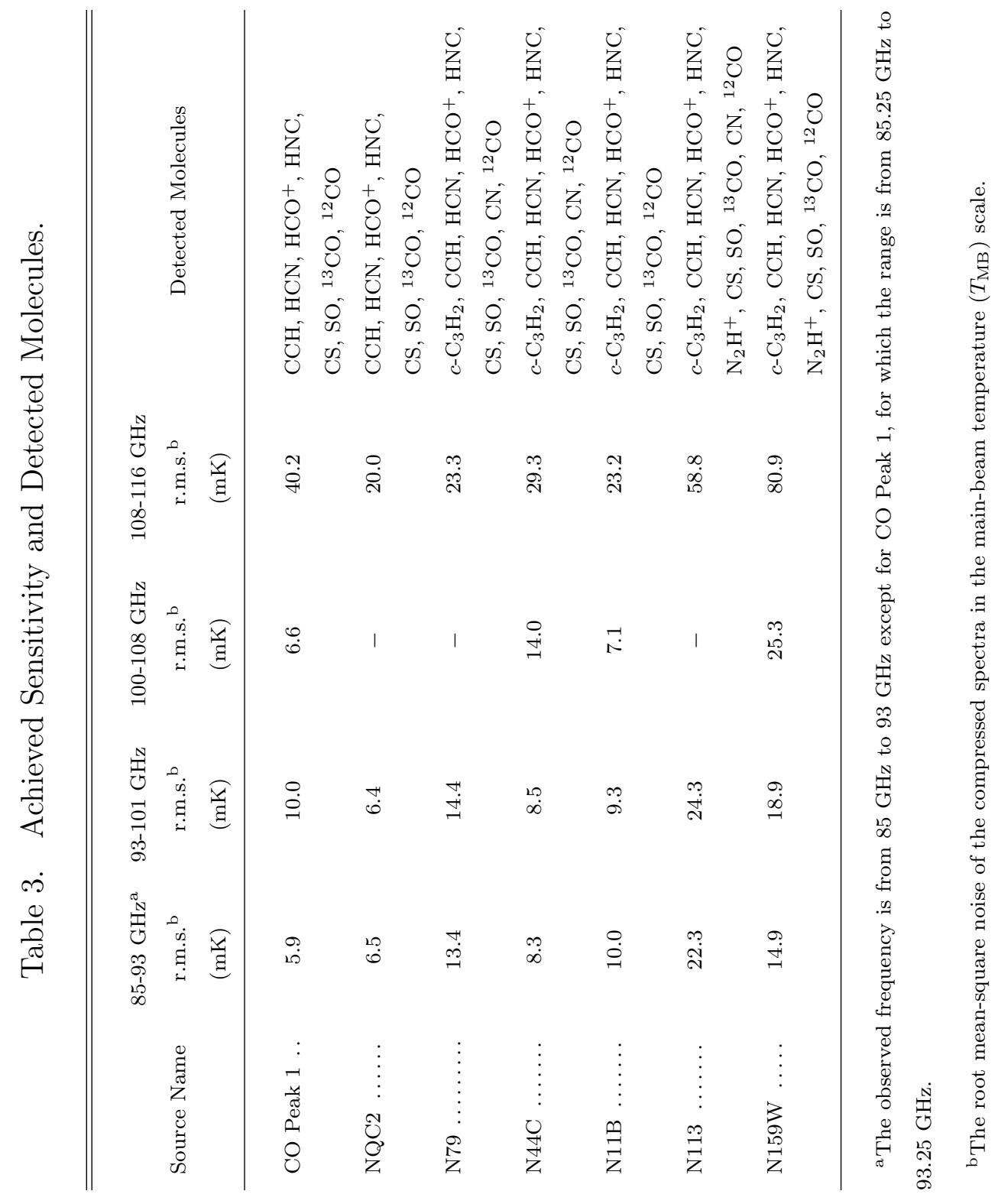




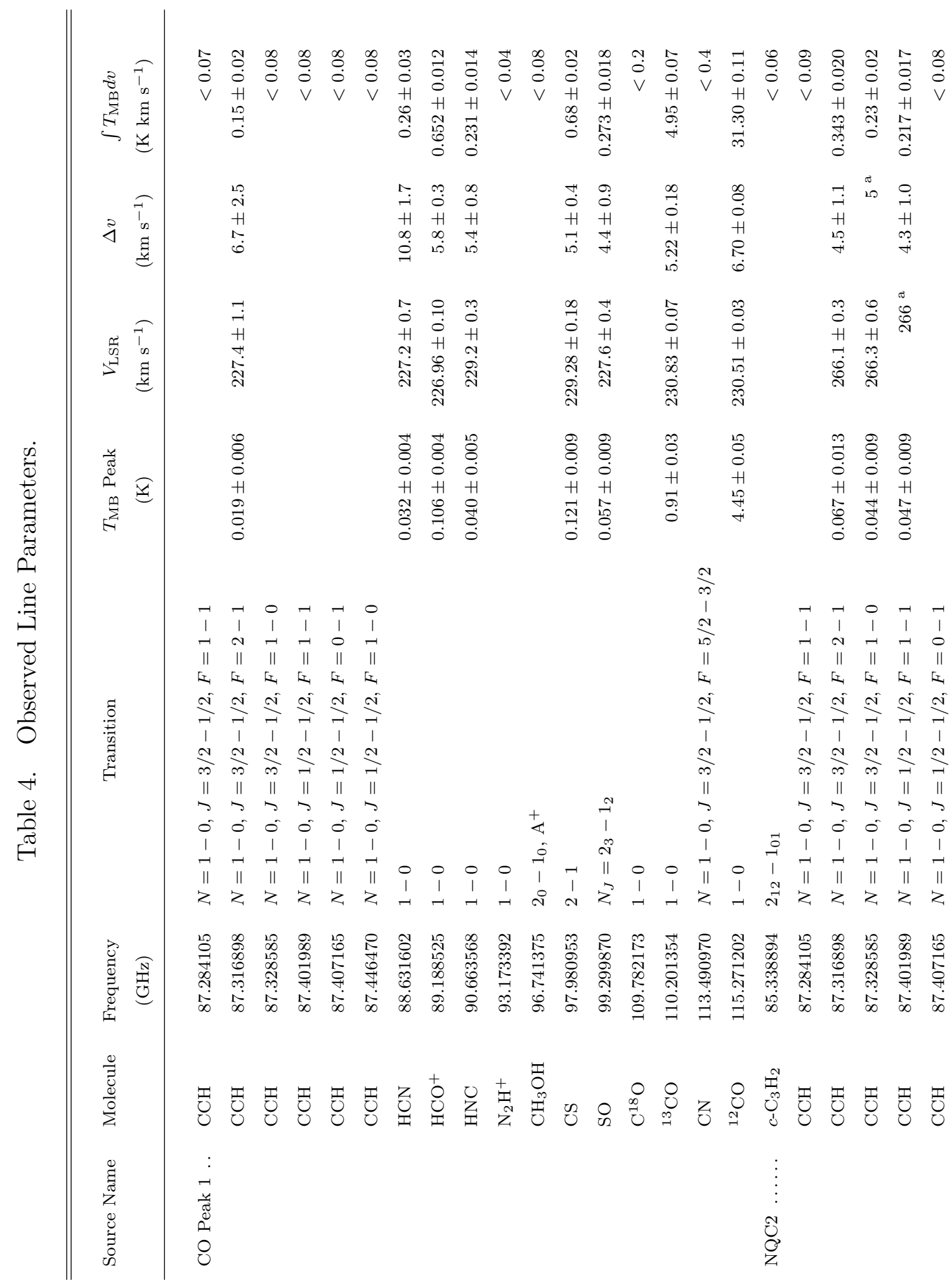




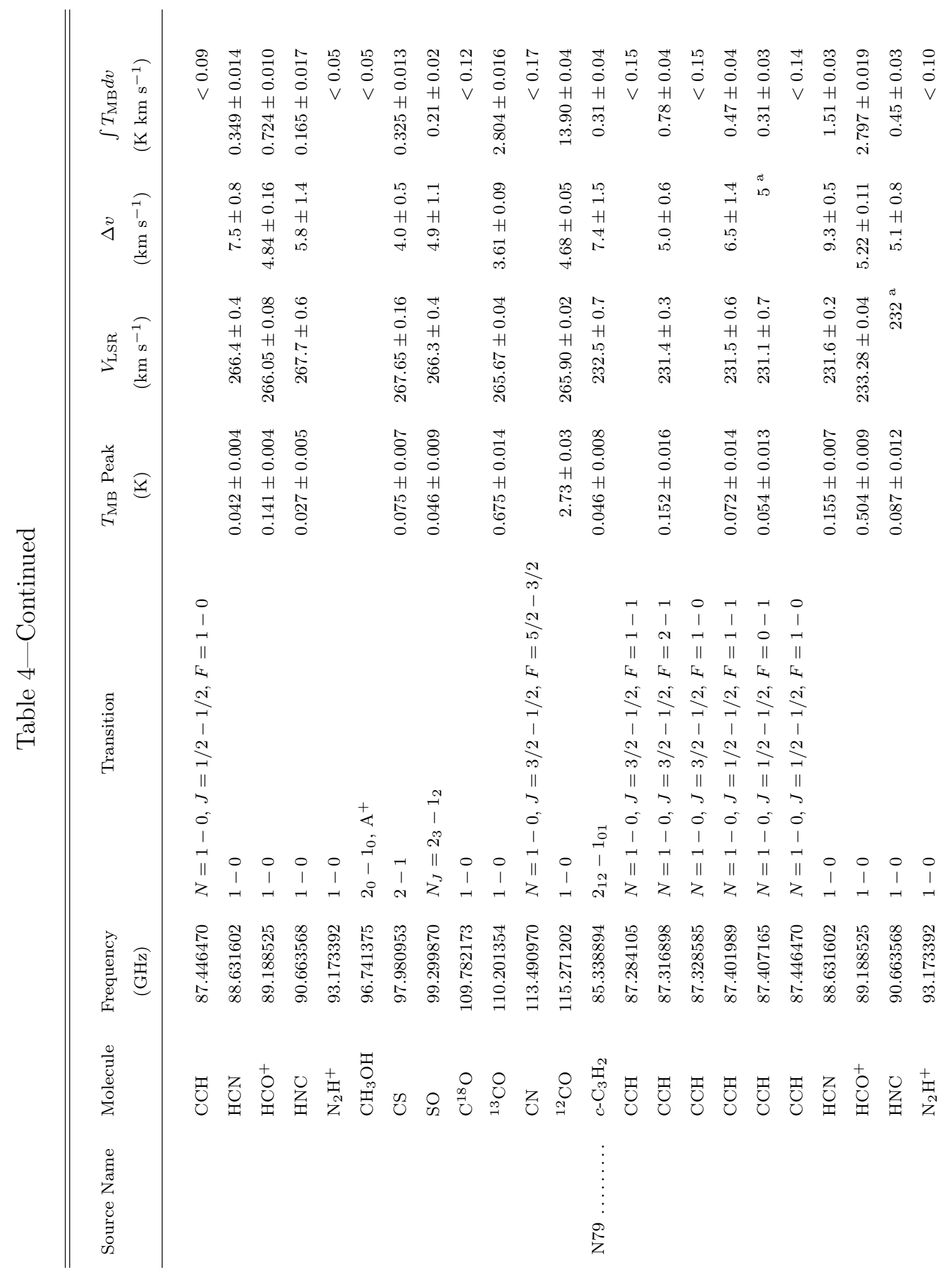




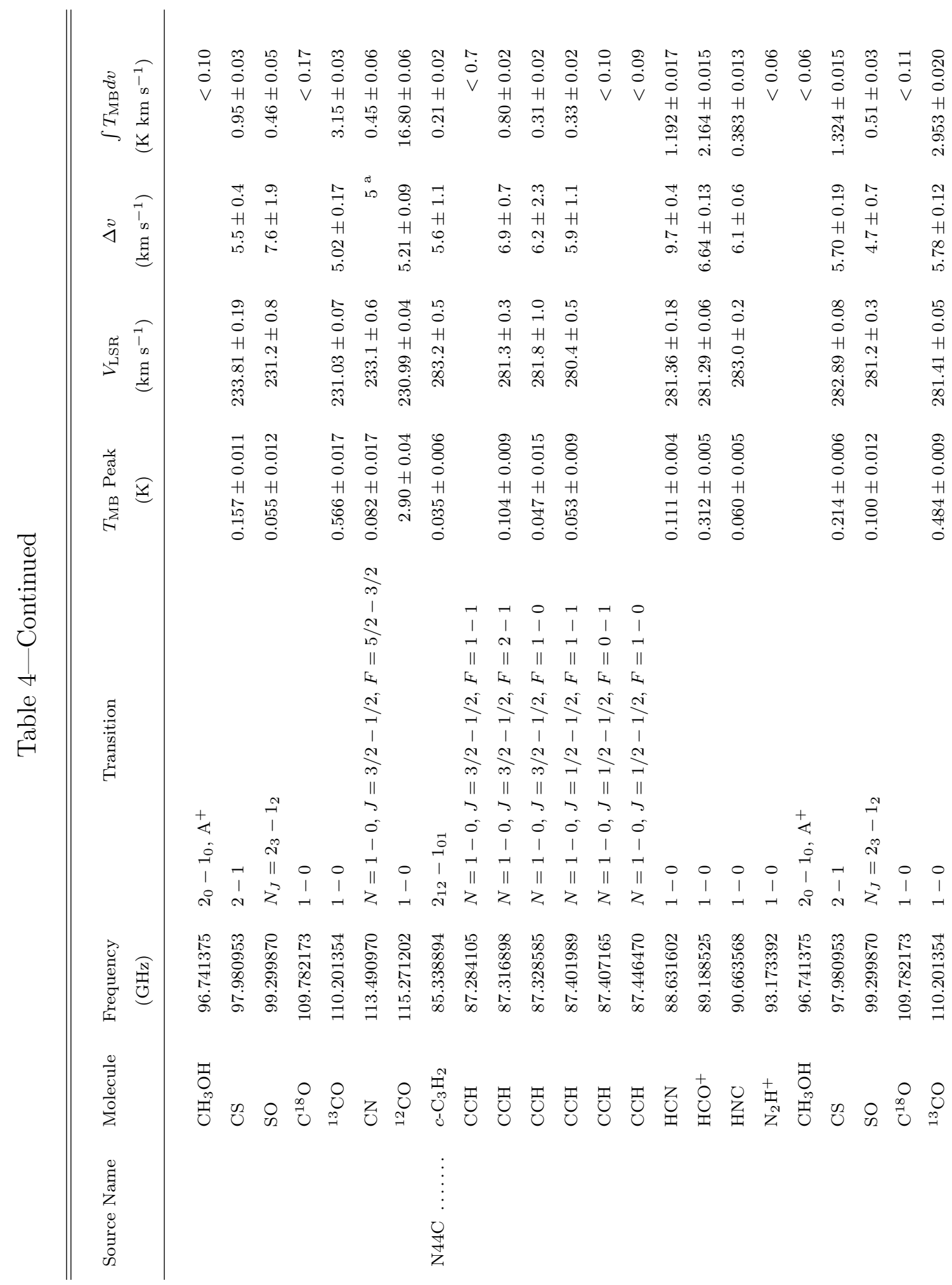




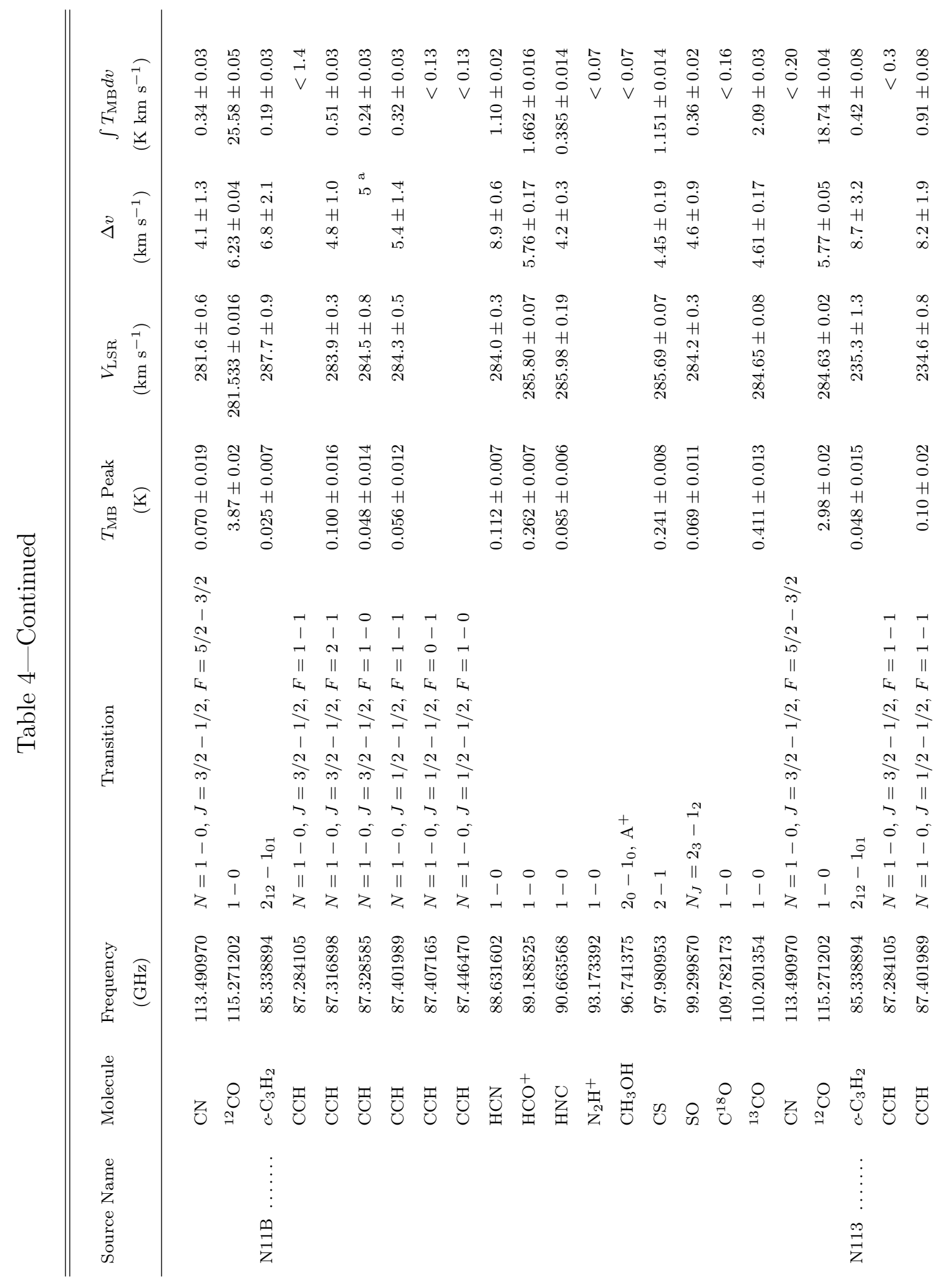




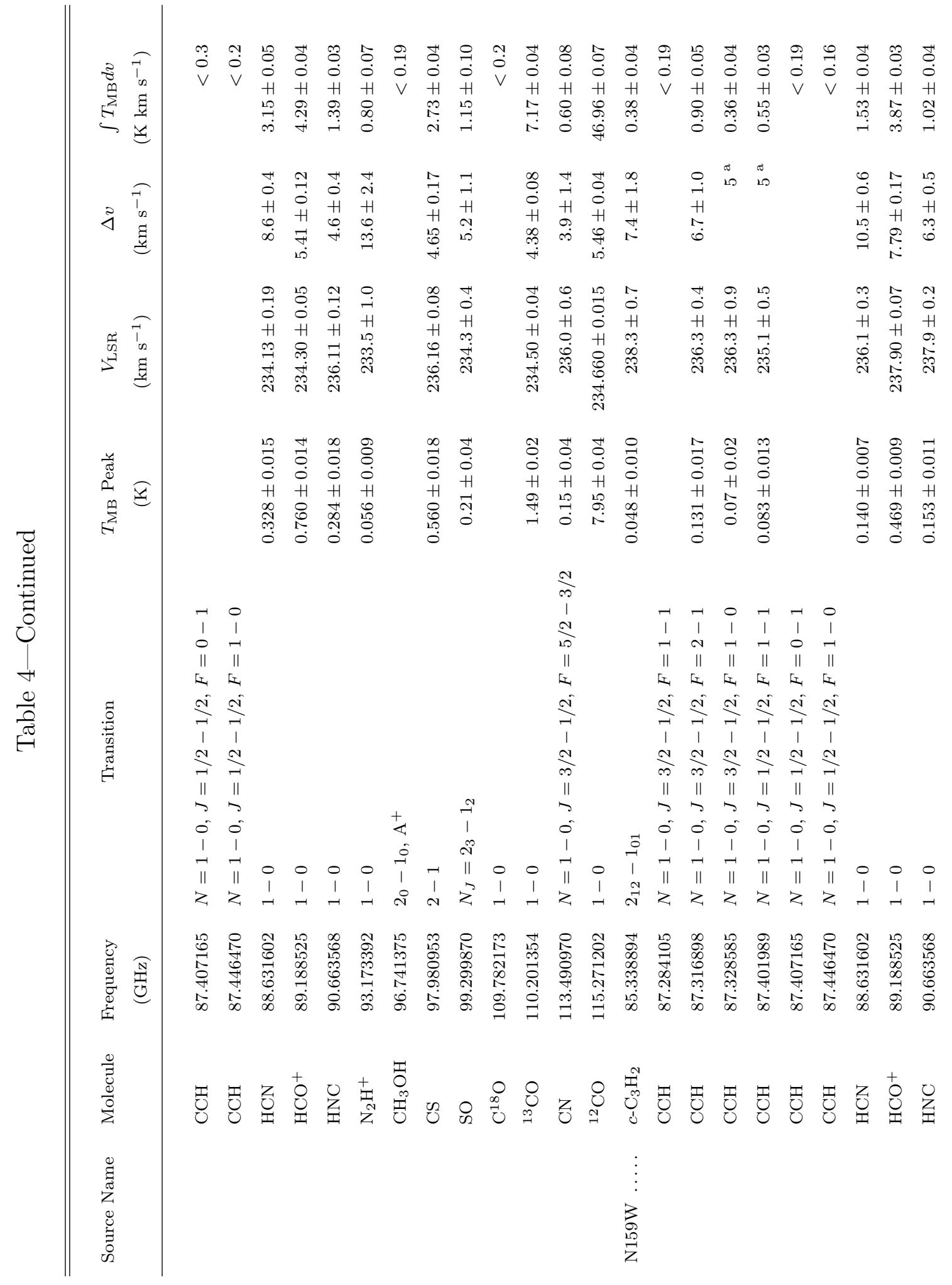




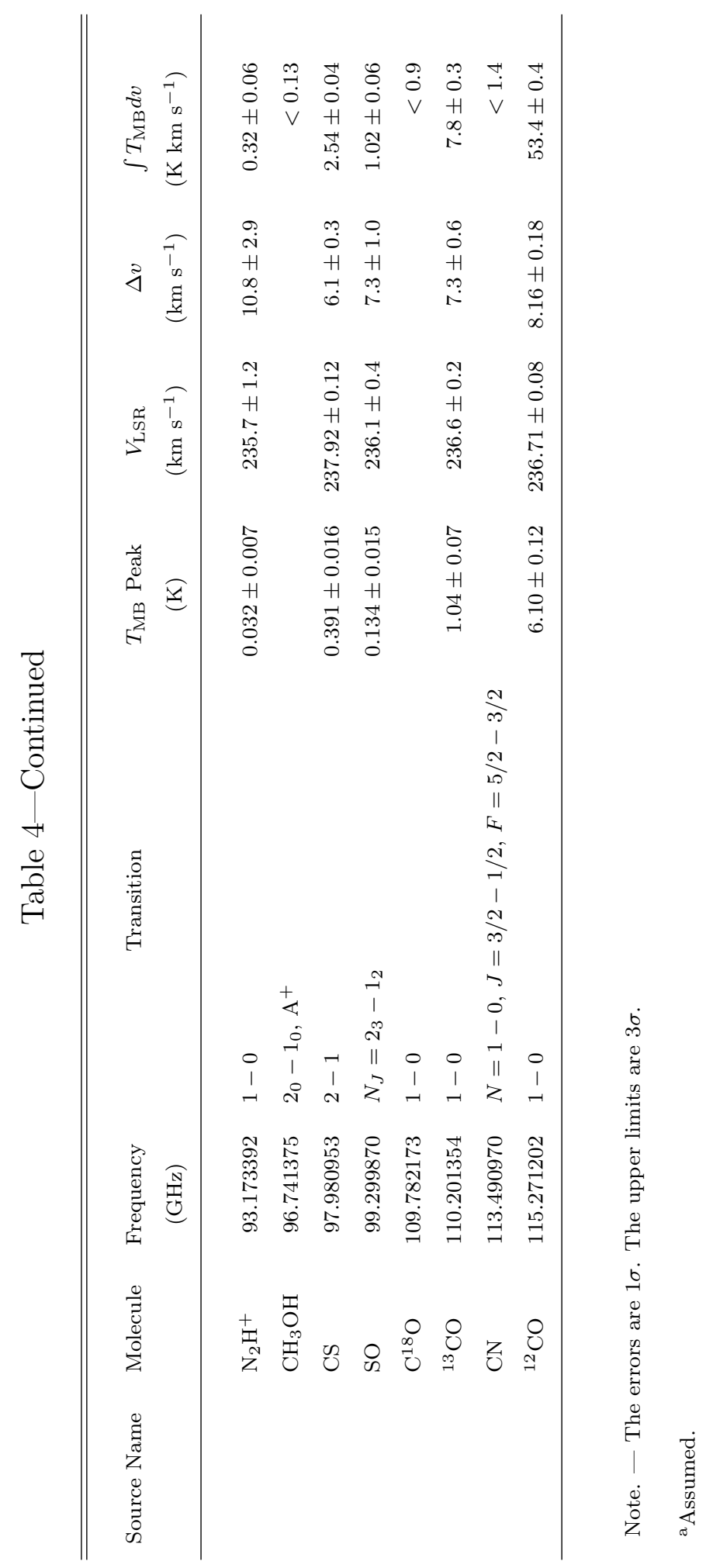


Table 5. Correlation Coefficients of Integrated Intensities of Molecules among the Seven Sources. ${ }^{a}$

\begin{tabular}{llllllll}
\hline \hline & & & & & & & \\
& $\mathrm{CCH}$ & $\mathrm{HCN}$ & $\mathrm{HCO}^{+}$ & $\mathrm{HNC}$ & $\mathrm{CS}$ & $\mathrm{SO}$ & ${ }^{13} \mathrm{CO}$ \\
\hline $\mathrm{CCH}$ & 1.000 & & & & & & \\
$\mathrm{HCN}$ & 0.985 & 1.000 & & & & & \\
$\mathrm{HCO}^{+}$ & 0.964 & 0.983 & 1.000 & & & & \\
$\mathrm{HNC}^{2}$ & 0.963 & 0.966 & 0.911 & 1.000 & & & \\
$\mathrm{CS}^{\mathrm{SO}}$ & 0.947 & 0.951 & 0.903 & 0.964 & 1.000 & & \\
${ }^{13} \mathrm{CO}$ & 0.981 & 0.971 & 0.933 & 0.987 & 0.978 & 1.000 & \\
\hline
\end{tabular}

aThe correlation coefficient $c$ is calculated as

$$
c=\frac{\sum\left(x_{i}-\bar{x}\right)\left(y_{i}-\bar{y}\right)}{\sqrt{\sum\left(x_{i}-\bar{x}\right)^{2} \sum\left(y_{i}-\bar{y}\right)^{2}}}
$$

where $x_{i}$ and $y_{i}$ are observed integrated intensities for the $i$-th source. $\bar{x}$ and $\bar{y}$ are the averages of $x_{i}$ and $y_{i}$, respectively. Correlation coefficients are higher than 0.9 except for the pairs including ${ }^{13} \mathrm{CO}$. 
Table 6. Derived Column Densities ${ }^{\mathrm{a}}$

\begin{tabular}{|c|c|c|c|c|c|c|c|c|c|c|}
\hline & \multicolumn{10}{|c|}{$T=10 \mathrm{~K}, n_{\mathrm{H}_{2}}=1 \times 10^{4} \mathrm{~cm}^{-3}$} \\
\hline & \multicolumn{3}{|c|}{ Translucent Cloud } & \multicolumn{7}{|c|}{ LMC } \\
\hline & CB17 & $\mathrm{CB} 24$ & CB228 & COP1 & NQC2 & N79 & $\mathrm{N} 44 \mathrm{C}$ & N11B & N113 & N159W \\
\hline$c-\mathrm{C}_{3} \mathrm{H}_{2}$ (ortho) & 84 & 3.8 & 5.4 & - & $<4.3$ & 26 & 15 & 12 & 32 & 27 \\
\hline $\mathrm{CCH}$ & 110 & 21 & 9.0 & 31 & 73 & 190 & 170 & 120 & 400 & 210 \\
\hline $\mathrm{HCN}$ & 180 & 32 & 24 & 16 & 15 & 79 & 56 & 52 & 170 & 79 \\
\hline $\mathrm{HCO}^{+}$ & 28 & 8.8 & 2.0 & 3.5 & 3.9 & 20 & 14 & 9.5 & 37 & 27 \\
\hline $\mathrm{HNC}$ & 110 & 11 & 9.1 & 4.1 & 3.0 & 9.0 & 7.2 & 7.3 & 34 & 22 \\
\hline $\mathrm{CH}_{3} \mathrm{OH}(\mathrm{A})$ & 6.2 & 2.6 & 8.0 & $<3.3$ & $<2.1$ & $<4.2$ & $<2.5$ & $<2.9$ & $<7.9$ & $<5.4$ \\
\hline $\mathrm{CS}$ & 76 & 22 & 12 & 28 & 13 & 40 & 59 & 53 & 170 & 130 \\
\hline $\mathrm{SO}$ & 34 & 33 & 38 & 18 & 16 & 30 & 35 & 23 & 85 & 73 \\
\hline \multirow[t]{4}{*}{${ }^{13} \mathrm{CO}$} & 7300 & 4800 & 3800 & 4700 & 2400 & 2700 & 2700 & 1800 & 7000 & 7700 \\
\hline & \multicolumn{10}{|c|}{$T=20 \mathrm{~K}, n_{\mathrm{H}_{2}}=1 \times 10^{4} \mathrm{~cm}^{-3}$} \\
\hline & \multicolumn{3}{|c|}{ Translucent Cloud } & \multicolumn{7}{|c|}{ LMC } \\
\hline & CB17 & $\mathrm{CB} 24$ & CB228 & $\mathrm{COP} 1$ & NQC2 & N79 & $\mathrm{N} 44 \mathrm{C}$ & N11B & N113 & N159W \\
\hline$c-\mathrm{C}_{3} \mathrm{H}_{2}$ (ortho) & 31 & 1.3 & 1.9 & - & $<1.8$ & 10 & 6.0 & 5.1 & 13 & 11 \\
\hline $\mathrm{CCH}$ & 52 & 11 & 4.8 & 16 & 39 & 99 & 93 & 62 & 260 & 110 \\
\hline $\mathrm{HCN}$ & 81 & 15 & 11 & 8.2 & 7.5 & 37 & 27 & 25 & 79 & 37 \\
\hline $\mathrm{HCO}^{+}$ & 13 & 4.4 & 1.1 & 2.1 & 2.4 & 11 & 7.9 & 5.6 & 19 & 15 \\
\hline $\mathrm{HNC}$ & 620 & 6.6 & 5.6 & 2.8 & 2.0 & 6.0 & 4.8 & 4.8 & 22 & 14 \\
\hline $\mathrm{CH}_{3} \mathrm{OH}(\mathrm{A})$ & 3.9 & 1.6 & 8.0 & $<2.2$ & $<1.4$ & $<2.7$ & $<1.6$ & $<1.9$ & $<5.1$ & $<3.5$ \\
\hline CS & 38 & 11 & 6.4 & 16 & 7.8 & 23 & 34 & 30 & 86 & 72 \\
\hline $\mathrm{SO}$ & 14 & 14 & 15 & 9.1 & 8.1 & 15 & 18 & 11 & 41 & 36 \\
\hline \multirow[t]{4}{*}{${ }^{13} \mathrm{CO}$} & 4800 & 3600 & 3200 & 5000 & 2600 & 3000 & 2900 & 2000 & 7100 & 8100 \\
\hline & \multicolumn{10}{|c|}{$T=30 \mathrm{~K}, n_{\mathrm{H}_{2}}=1 \times 10^{4} \mathrm{~cm}^{-3}$} \\
\hline & \multicolumn{3}{|c|}{ Translucent Cloud } & \multicolumn{7}{|c|}{ LMC } \\
\hline & $\mathrm{CB} 17$ & $\mathrm{CB} 24$ & CB228 & $\mathrm{COP} 1$ & $\mathrm{NQC2}$ & N79 & $\mathrm{N} 44 \mathrm{C}$ & N11B & N113 & N159W \\
\hline$c-\mathrm{C}_{3} \mathrm{H}_{2}$ (ortho) & 18 & 0.83 & 1.1 & - & $<1.1$ & 6.7 & 3.8 & 3.3 & 8.2 & 6.9 \\
\hline
\end{tabular}


Table 6-Continued

\begin{tabular}{lcccccccccc}
\hline \hline $\mathrm{CCH}$ & 41 & 8.9 & 3.9 & 13 & 31 & 80 & 75 & 50 & 210 & 92 \\
$\mathrm{HCN}$ & 58 & 1.1 & 7.7 & 6.1 & 5.6 & 24 & 20 & 18 & 57 & 27 \\
$\mathrm{HCO}^{+}$ & 9.9 & 3.3 & 0.91 & 1.8 & 2.0 & 8.8 & 6.4 & 4.6 & 15 & 12 \\
$\mathrm{HNC}$ & 52 & 5.6 & 4.8 & 2.5 & 1.8 & 5.3 & 4.3 & 4.2 & 18 & 12 \\
$\mathrm{CH}_{3} \mathrm{OH}(\mathrm{A})$ & 3.4 & 1.4 & 4.3 & $<1.9$ & $<1.2$ & $<2.4$ & $<1.4$ & $<1.7$ & $<4.5$ & $<3.1$ \\
$\mathrm{CS}$ & 28 & 8.2 & 4.9 & 13 & 6.2 & 18 & 24 & 24 & 66 & 56 \\
$\mathrm{SO}$ & 10 & 9.9 & 11 & 7.0 & 6.3 & 11 & 13 & 8.8 & 31 & 27 \\
13 & 5100 & 3900 & 2500 & 5700 & 2900 & 3400 & 3300 & 2300 & 8100 & 9200 \\
\hline
\end{tabular}

${ }^{a}$ In units of $10^{12} \mathrm{~cm}^{-2}$. The column densities are derived by the statistical equilibrium calculation (See text). Results are given for an $\mathrm{H}_{2}$ density of $1 \times 10^{4} \mathrm{~cm}^{-3}$ and three different temperatures. 
Table 7. Column Density Ratios of Relative to $\mathrm{HCO}^{+}$.

\begin{tabular}{|c|c|c|c|c|c|}
\hline & $\mathrm{CCH}$ & $\mathrm{HCN}$ & $\mathrm{HNC}$ & CS & $\mathrm{SO}$ \\
\hline CO Peak 1 & $8.3_{-1.3}^{+1.7}$ & $3.6_{-1.4}^{+1.3}$ & $1.2_{-0.2}^{+0.3}$ & $7.4_{-1.2}^{+1.0}$ & $4.6_{-0.7}^{+0.7}$ \\
\hline NQC2 & $17.2_{-3.0}^{+3.7}$ & $3.0_{-1.1}^{+1.0}$ & $0.8_{-0.1}^{+0.1}$ & $3.1_{-0.5}^{+0.2}$ & $3.6_{-0.7}^{+0.6}$ \\
\hline N79 & $9.9_{-2.2}^{+3.1}$ & $3.2_{-0.9}^{+0.8}$ & $0.5_{-0.1}^{+0.1}$ & $2.0_{-0.1}^{+0.1}$ & $1.4_{-0.5}^{+0.4}$ \\
\hline $\mathrm{N} 44 \mathrm{C}$ & $12.7_{-2.7}^{+3.3}$ & $3.2_{-1.1}^{+0.9}$ & $0.5_{-0.1}^{+0.1}$ & $4.1_{-0.5}^{+0.4}$ & $2.3_{-0.4}^{+0.4}$ \\
\hline N11B & $12.1_{-2.3}^{+3.1}$ & $4.3_{-1.6}^{+1.7}$ & $0.9_{-0.1}^{+0.2}$ & $5.2_{-0.8}^{+0.6}$ & $2.2_{-0.4}^{+0.4}$ \\
\hline N113 & $13.9_{-4.4}^{+5.7}$ & $3.9_{-1.1}^{+0.9}$ & $1.0_{-0.2}^{+0.2}$ & $4.3_{-0.4}^{+0.3}$ & $2.3_{-0.5}^{+0.6}$ \\
\hline N159W & $8.2_{-1.9}^{+2.6}$ & $2.3_{-0.7}^{+0.6}$ & $0.9_{-0.1}^{+0.2}$ & $4.6_{-0.8}^{+0.4}$ & $2.5_{-0.5}^{+0.6}$ \\
\hline LMC average & $11.7 \pm 3.0$ & $3.4 \pm 0.6$ & $0.8 \pm 0.2$ & $4.4 \pm 1.6$ & $2.7 \pm 1.0$ \\
\hline $\mathrm{N} 113^{\mathrm{a}}$ & - & 1.6 & 0.6 & 6.3 & 4.0 \\
\hline N159W & 19.3 & 0.9 & 0.3 & 1.4 & 1.6 \\
\hline N159S ${ }^{b}$ & 30.0 & 1.0 & 0.3 & 5.0 & 5.0 \\
\hline $\mathrm{N} 160^{\mathrm{b}}$ & 15.1 & 1.2 & - & 1.0 & 1.7 \\
\hline 30 Dor- $10^{\mathrm{b}}$ & 18.2 & 0.7 & 0.2 & 0.9 & 0.7 \\
\hline 30 Dor- $27^{\mathrm{b}}$ & 38.7 & 1.2 & - & 4.8 & 2.7 \\
\hline Model of the LMC (L1) ${ }^{\mathrm{c}}$ & 3.4 & 2.3 & 1.3 & - & - \\
\hline Model of the LMC $(\mathrm{L} 2)^{\mathrm{c}}$ & 4.0 & 0.10 & 0.09 & - & - \\
\hline Model of Milky Way $(\mathrm{G})^{\mathrm{c}}$ & 5.2 & 5.7 & 3.1 & - & - \\
\hline Milky Way (38 translucent clouds) ${ }^{\mathrm{d}}$ & - & 9.3 & 2.2 & 8.1 & 4.5 \\
\hline Milky Way (except CB17) ${ }^{\mathrm{d}}$ & - & 19.3 & 3.5 & 9.5 & 10.3 \\
\hline Milky Way (CB17, CB24, CB228) & 4.2 & 6.1 & 3.4 & 5.1 & 8.4 \\
\hline $\mathrm{M} 51^{\mathrm{e}}$ & 6.7 & 9.6 & 1.7 & 1.9 & 0.4 \\
\hline
\end{tabular}

Wang et al. (2009).

$\mathrm{b}$ Heikkilä et al. (1999).

'Millar \& Herbst (1990).

${ }^{\mathrm{d}}$ Derived from the statistical equilibrium calculation based on Turner (1995a b, 1996); Turner et al. (1997, 1999).

e Derived from the statistical equilibrium calculation based on Watanabe et al. (2014). 